\title{
Bubble merger model for the nonlinear Rayleigh- Taylor instability driven by a strong blast wave
}

\author{
Aaron R.Miles - Lawrence Livermore National \\ Laboratory
}

Technical Report

U.S. Department of Energy

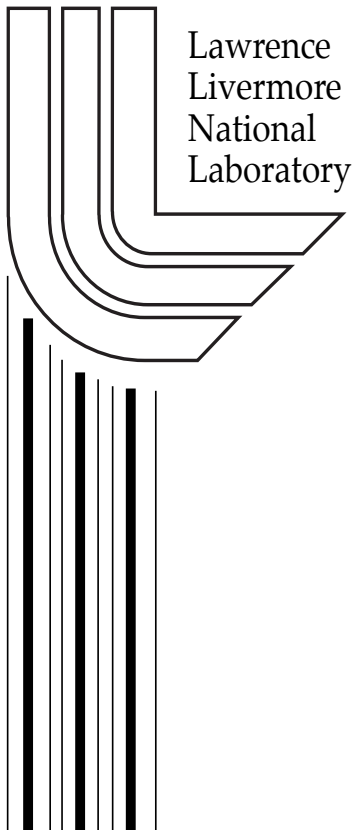

March 2004 


\section{DISCLAIMER}

This document was prepared as an account of work sponsored by an agency of the United States Government. Neither the United States Government nor the University of California nor any of their employees, makes any warranty, express or implied, or assumes any legal liability or responsibility for the accuracy, completeness, or usefulness of any information, apparatus, product, or process disclosed, or represents that its use would not infringe privately owned rights. Reference herein to any specific commercial product, process, or service by trade name, trademark, manufacturer, or otherwise, does not necessarily constitute or imply its endorsement, recommendation, or favoring by the United States Government or the University of California. The views and opinions of authors expressed herein do not necessarily state or reflect those of the United States Government or the University of California, and shall not be used for advertising or product endorsement purposes.

This work was performed under the auspices of the U. S. Department of Energy by the University of California, Lawrence Livermore National Laboratory under Contract No. W-7405-Eng-48.

This report has been reproduced directly from the best available copy.

Available to DOE and DOE contractors from the

Office of Scientific and Technical Information

P.O. Box 62, Oak Ridge, TN 37831

Prices available from (423) 576-8401

http: / / apollo.osti.gov/bridge/

Available to the public from the National Technical Information Service

U.S. Department of Commerce 5285 Port Royal Rd., Springfield, VA 22161 http:/ / www.ntis.gov/

OR

Lawrence Livermore National Laboratory Technical Information Department's Digital Library http:/ / www.llnl.gov/tid/Library.html 


\title{
Bubble merger model for the nonlinear Rayleigh-Taylor instability driven by a strong blast wave
}

\author{
A. R. Miles \\ Lawrence Livermore National Laboratory, Livermore, CA 94550 \\ and University of Maryland, College Park, MD 20741
}

From a dissertation to be submitted to the Graduate School, University of Maryland, by Aaron Miles in partial fulfillment of the requirements for the Ph.D. Degree in Physics.

A bubble merger model is presented for the nonlinear evolution of the Rayleigh-Taylor instability driven by a strong blast wave. Single bubble motion is determined by an extension of previous buoyancy-drag models extended to the blast wave driven case, and a simple bubble merger law in the spirit of the Sharp-Wheeler model allows for the generation of larger scales. The blast wave driven case differs in several respects from the classical case of incompressible fluids in a uniform gravitational field. Because of material decompression in the rarefaction behind the blast front, the asymptotic bubble velocity and the merger time depend on time as well as the transverse scale and the drive. For planar blast waves, this precludes the emergence of a self-similar regime independent of the initial conditions. With higher-dimensional blast waves, divergence restores the properties necessary for the establishment of the self-similar state, but its establishment requires a very high initial characteristic mode number and a high Mach number for the incident blast wave.

${ }^{a)}$ Electronic mail: miles15@1lnl.gov 


\section{Introduction}

The basic Rayleigh-Taylor (RT) instability criterion, ${ }^{1,2}$ neglecting certain potentially stabilizing factors such as surface tension ${ }^{3}$, is the existence of anti-parallel components of pressure and density gradients $(\nabla \mathrm{P} \bullet \nabla \rho<0)$. When this condition is met at an interface between two materials with density ratio $\eta<1$, perturbations on the interface will grow in time. In the inviscid limit, the instability develops exponentially while the perturbations remain small (during the linear phase) with a growth rate given by ${ }^{4}$

$$
\gamma=\sqrt{\frac{k g A}{1+k L}}
$$

where $\mathrm{k}$ is the perturbation wavenumber, $\mathrm{g}$ is the acceleration, $\mathrm{A}=(1-\eta) /(1+\eta)$ is the Atwood number, and $\mathrm{L}$ is the density gradient scale length at the interface. At later times, initially sinusoidal perturbations grow into spikes of heavier fluid "falling" into lighter fluid and bubbles of lighter fluid "rising" into heavier fluid. For A = 1, the bubbles rise with constant (terminal) velocity while spikes fall with constant acceleration in the nonlinear regime. ${ }^{5,6}$ When $\mathrm{A}<1$, the spike eventually also reaches terminal velocity. ${ }^{6}$

The RT instability criterion can also be satisfied at a material interface through which a blast wave has been transmitted from a heavier to a lighter fluid. ${ }^{8}$ As is illustrated in Fig. 1, since the pressure behind a blast wave is always falling in time at any fixed point and in distance behind the shock front (at least in the self-similar regime ${ }^{9}$ ), an 
interface generally becomes RT unstable when it transmits a blast wave down a density gradient (ie from more dense to less dense material).

Shock and blast waves are common participants in astrophysical processes. ${ }^{10}$ In a core-collapse supernova, for example, the sudden release of an enormous amount of energy at the star's core drives a strong blast wave that propagates out through layers of progressively less dense matter. ${ }^{11,12}$ As the interfaces between these layers subsequently decelerate in the expansion fan behind the blast front, they are RT unstable. ${ }^{8}$ The potential significance of this phenomenon was realized with observations of SN1987A, when it was found that spherically-symmetric explosion models failed to correctly predict the velocity and arrival time at the surface of heavy elements originating from the star's central regions. It has been suggested that the discrepancy results from the failure of the 1D models to account for the turbulent (the Reynolds number has been estimated ${ }^{13}$ to be of order $10^{10}$ ) RT mixing that is certainly occurring at unstable interfaces. ${ }^{11,12,14,15}$

A great deal of experimental, theoretical, and computational work has been directed towards understanding the evolution of RT unstable systems, and much progress has been made in the last fifty years. Much of this effort has focused on the classical case of incompressible fluids in a uniform acceleration field. For systems driven by strong blast waves, such as core-collapse supernovae and the high-energy-density laser-driven experiments $^{16-24}$ designed to study them, the acceleration is strongly time-dependent and the flow is compressible. Consequently, ideas and observations pertaining to classical RT systems do not necessarily apply. The purpose of this paper is to examine how models of nonlinear RT growth should be modified when applied to blast-wave-driven systems and 
what implications these differences have on self-similarity, loss of initial conditions, and transition to turbulence.

We begin by reviewing the stages of classical RT instability growth, focusing on the nonlinear regime, and describe the types of models commonly used to predict the evolution of multimode interfaces. We use a simple version of the Sharp-Wheeler bubble merger model ${ }^{25,26}$ to illustrate the loss of memory of initial conditions and the hypothesized establishment of a self-similar regime, and discuss their relationship to the system's transition to turbulence. After a brief review of relevant aspects of blast wave theory, we outline in more detail the peculiarities of blast-wave-driven RT and present an appropriately generalized buoyancy-drag model. We discuss bubble merger for the blastwave-driven case and the statistical-mechanical merger model resulting from combining it with the single-mode buoyancy-drag model.

For planar blast-waves, the onset of self-similar instability growth and the loss of memory of initial conditions do not follow from the model as they do in the classical case. Instead, the ratio of mix width to dominant transverse scale grows slowly in time during what we call a quasi-self-similar regime.

Self-similarity and loss of memory of initial conditions are possible for divergent systems, but require very high initial characteristic mode numbers and high incident Mach numbers. This requirement has serious implications for supernovae. Initial conditions predicted by recent stellar calculations ${ }^{27.28}$ suggest that initial mode numbers present in supernova progenitors are not high enough to reach the self-similar regime. Instead, the late-time instability evolution would depend on the initial perturbation spectrum. 
Finally, we include a generalization of the model for interfaces driven by nonideal blast waves and make some comparisons with simulations, experiments, and observations.

\section{Classical case}

In its most basic formulation, the Rayleigh-Taylor instability describes the evolution of an interface separating a heavier (more dense) fluid supported by a lighter (less dense) fluid in a uniform acceleration field. In the linear approximation, which is valid for ka $<1$, pertubation growth is exponential with a growth rate given by Eq. (1). When the amplitude becomes comparable to the wavelength, the linear approximation breaks down. Buoyancy-drag models treat bubbles of lighter fluid and spikes of heavier fluid as the fundamental nonlinear objects. The model of Oron et al, ${ }^{29}$ which follows earlier work by Youngs ${ }^{30}$ and Dimonte and Schneider, ${ }^{31}$ predicts that $2 \mathrm{D}$ bubbles "rise" with a velocity determined by

$$
\left(\rho_{b}+C_{a} \rho_{s}\right) \frac{d u_{b}(t)}{d t}=\left(\rho_{s}-\rho_{b}\right) g(t)-\frac{C_{D}}{\lambda} \rho_{s} u_{b}^{2}
$$

The equation for the spike velocity is obtained by simply interchanging spike and bubble densities. The added mass coefficient $C_{a}$ equals 2 for $2 \mathrm{D}$ perturbations and 1 for $3 \mathrm{D}$ perturbations, while the drag coefficient $C_{D}$ is $6 \pi$ for $2 D$ perturbations and $2 \beta_{1} \approx 7.66 \approx$ $1.22 \times 2 \pi$, where $\beta_{1}$ is the first zero of the first-order Bessel function, for $3 \mathrm{D}$ perturbations. Some authors, including Oron et al., ${ }^{29}$ use $C_{D}=2 \pi$ for $3 \mathrm{D}$ bubbles. These drag coefficients, originally derived by Layzer ${ }^{32}$ following earlier work by Davies and 
Taylor, ${ }^{33}$ apply to $\mathrm{A}=1$ bubbles rising in tubes of diameter $\lambda$ or $2 \mathrm{D}$ flow between parallel plates. In either case, $\lambda$ is twice the transverse scale of the bubble. Since spikes at $A=1$ are infinitely narrow, the transverse scale of the bubble is equal to the wavelength of a periodic array of bubbles. As A decreases below unity, however, the width of the spikes increases until, at $\mathrm{A}=0$, there is no distinction between spikes and bubbles. Rather than making the transverse scale in Eq. (2a) a function of the perturbation wavelength and Atwood number, we instead redefine the drag coefficients so that

$$
\begin{aligned}
& C_{D}{ }^{2 D}=\left\{\begin{array}{ll}
3 \pi(1+A) & \text { for bubbles } \\
3 \pi(1-A) & \text { for spikes }
\end{array}\right\} \\
& C_{D}{ }^{3 D}=\left\{\begin{array}{ll}
\beta_{1}(1+A) & \text { for bubbles } \\
\beta_{1}(1-A) & \text { for spikes }
\end{array}\right\}
\end{aligned}
$$

With this definition, $\lambda$ is always the perturbation wavelength. Because of KelvinHelmholtz $(\mathrm{KH})$ rollup at the spike tips for $\mathrm{A}<1$, the actual spike width will be greater than that implied by (2b). The same is true for bubbles at low Atwood number. In those cases where KH effects have a significant impact on the spike and/or bubble width, the drag coefficients should be adjusted accordingly.

Strictly speaking, the values in (2a) apply to single bubbles that are laterally confined or periodic bubble arrays. For chaotic bubble fronts, experiments ${ }^{34}$ and simulations $^{35}$ suggest that the drag coefficients should be smaller by a factor of about $6 \pi$, corresponding to asymptotic velocities that are higher by a factor of about 2.5. Glimm and Li have suggested that this is because the leading bubbles in chaotic arrays are laterally less confined by their neighbors then those in periodic arrays. ${ }^{26}$

Equation (2a) is just Newton's second law, where the inertial term on the left of the equals sign contains an added mass coefficient $\mathrm{C}_{\mathrm{a}}$ and the two forces (per unit 
volume) on the right-hand side are buoyancy and drag. Terminal velocity is attained when the two forces balance one another, and the resulting asymptotic velocity is

$$
u_{b, s}^{a s y m}=\sqrt{\frac{\left|1-\eta_{b, s}\right|}{C_{D}} L g} \equiv C_{b, s} \sqrt{L g},
$$

where $\eta_{\mathrm{b}, \mathrm{s}}=\rho_{\mathrm{b}, \mathrm{s}} / \rho_{\mathrm{s}, \mathrm{b}}$. In what follows, we typically drop the spike and bubble subscripts with the understanding that the merger models describe mergers between bubbles rather than spikes. Merger models are based on the fact that larger bubbles rise (or grow) faster than smaller bubbles and the observation that smaller bubbles "merge" to form larger bubbles. Statistical mechanics merger models for the Rayleigh-Taylor instability describe the evolution of a bubble size distribution function $g(\lambda, t)$, which gives the number of bubbles in the system with transverse sizes between $\lambda$ and $\lambda+\mathrm{d} \lambda$ at time $\mathrm{t}^{26,36}$ Such models are built out of two main components. The first component is a model for the velocity of a single bubble, such as Eq. (2) or, more typically, Eq. (3), and the second is a rule for the merger of two neighboring bubbles. Merger rates have been obtained from theory, ${ }^{26,32,36-39}$ simulation, ${ }^{26,40}$ and experiments. ${ }^{26}$ In the very simplest model, the merger rate is a constant independent of the size of the bubbles involved. ${ }^{36}$ In a more reasonable approach, which we will adopt, two neighboring bubbles are said to have merged when the larger of the two has risen above the smaller bubble by a constant fraction of the smaller bubble's transverse size. ${ }^{26,37}$ In either case, the smaller bubble, observed experimentally ${ }^{34}$ to be "washed down stream", is removed from the ensemble. In $2 \mathrm{D}$, the diameter of the surviving bubble is equal to the sum of the diameters of the two premerger bubbles. In 3D, area rather than diameter is the conserved quantity.

The evolution of the bubble-size distribution $\mathrm{g}(\mathrm{L}, \mathrm{t})$ is given by ${ }^{36}$ 
$N(t) \frac{d g(L, t)}{d t}=-2 g(L, t) \int_{0}^{\infty} g\left(L^{\prime}, t\right) \omega\left(L, L^{\prime}\right) d L^{\prime}+\int_{0}^{\infty} g\left(L^{\prime}, t\right) g\left(L-L^{\prime}, t\right) \omega\left(L^{\prime}, L-L^{\prime}\right) d L^{\prime}$

where the total number of bubbles $N(t)=\int_{0}^{\infty} g(L, t) d L$ and the merger rate $\omega\left(\mathrm{L}, \mathrm{L}^{\prime}\right)$ is inversely proportional to the time interval required for a merger between two adjacent bubbles with transverse scales L and L'. The first term on the right-hand side is the rate at which bubbles of size L are lost in merger event, and the second term gives the rate at which bubbles of size L are generated.

It has been proposed that, at least for the classical case, an RT-unstable system tends to approach a self-similar (or scale-invariant) regime independent of the initial conditions after several generations of bubble merger above the largest significant scales present in the initial spectrum. ${ }^{41}$ This is true of the model given in Eq. (4) for a wide range of merger rates, and is also supported by a large body of experimental, ${ }^{34}$ theoretical, ${ }^{37,42}$ and computational ${ }^{41}$ work. Late-time independence of initial conditions in hydrodynamically-unstable systems means that two interfaces, even if characterized by wildly different perturbation spectra at time zero, will eventually reach a regime in which their perturbation amplitudes, velocities, and statistical spectral properties (or bubble size distribution functions) will become equalized. Strictly speaking, we require that the relative difference between the two systems vanishes in the limit that t goes to infinity. The instability evolution is self-similar if the shape of the bubble distribution function does not change in time except for a linear multiplier that increases in proportion with the bubble size expectation value. In the self-similar regime, the mix width $\mathrm{h}(\mathrm{t})$ grows in proportion to the characteristic bubble size (the bubble size expectation value).

To illustrate the mechanism by which initial conditions might be erased in the RT 
instability, consider the following toy model of bubble merger: Assume there is only one bubble size (L) at any time $t$, and that $\mathrm{L}_{0}$ is the bubble size at time zero. Then, at each merger, the bubble size is doubled, so we have

$$
\mathrm{L}(\mathrm{t})=2^{\mathrm{N}(\mathrm{t})} \mathrm{L}_{0},
$$

where $N(t)$ is the number of bubbles of size $L$ at time t. Now $N(t)$ is determined by the requirement that

$$
\mathrm{t}=\tau\left(\mathrm{L}_{0}\right)+\tau\left(2 \mathrm{~L}_{0}\right)+\ldots+\tau\left(2^{\mathrm{N}(t)} \mathrm{L}_{0}\right),
$$

where $\tau(L)$ is the merger time for a bubbles of size $L$. If we had $\tau(L)=\tau$ independent of $\mathrm{L}$, then $\mathrm{N}(\mathrm{t})=\mathrm{t} / \tau$, and so $\mathrm{L}$ would grow exponentially in time. Loss of initial conditions is possible because the merger rate is not independent of bubble size. Instead, larger bubbles take longer to merge than smaller bubbles. For classical RT, dimensional analysis requires that ${ }^{25}$

$$
\tau(L) \propto \sqrt{L / g} .
$$

The terminal velocity for bubbles of size L (and spikes for $\mathrm{A}<1$ ) is given by Eq. (3). The coefficient $\mathrm{C}$ depends on the dimension of the perturbation and the Atwood number. Note that Eq. (7) says that the bubble merger time is proportional to the time it takes the bubble to rise by one bubble diameter. We define the dimensionless constant $\mathrm{C}_{2}$ as the fraction of a bubble diameter the bubble must rise before merger takes place, so that

$$
\tau(L)=\left(C_{2} / C\right) \sqrt{L / g}=\left(C_{2} / C\right) \sqrt{L_{0} / g} 2^{i / 2},
$$

where $\mathrm{i}$ is the bubble generation number. Requirement (6) then becomes

$$
t=\frac{C_{2}}{C} \sqrt{\frac{L_{0}}{g}} \sum_{i=0}^{N} 2^{i / 2}=\frac{C_{2}}{C} \sqrt{\frac{L_{0}}{g}} \frac{\sqrt{2} 2^{N / 2}-1}{\sqrt{2}-1},
$$

from which it follows that 


$$
N(t)=2 \log \left[\frac{C}{C_{2}}(\sqrt{2}-1) \sqrt{\frac{g}{L_{0}}} t+1\right]-1 .
$$

Inserting this into (5), we finally get

$$
L(t)=\left[\frac{C}{C_{2}}\left(1-\frac{1}{\sqrt{2}}\right) \sqrt{g} t+\sqrt{\frac{L_{0}}{2}}\right]^{2} .
$$

For times such that the inequality

$$
t>>\frac{C_{2}}{C} \sqrt{\frac{L_{0}}{g}} \equiv \tau\left(L_{0}\right),
$$

is satisfied, the first term in the square brackets dominates, and we get the limiting result

$$
L(t) \rightarrow\left[\frac{C}{C_{2}}\left(1-\frac{1}{\sqrt{2}}\right)\right]^{2} g t^{2}
$$

That is, the dependence on initial conditions (the initial bubble size $\mathrm{L}_{0}$ ) is lost at times long compared to the first generation bubble merger time. Consequently, $\mathrm{gt}^{2}$ is the only transverse scale remaining in the problem.

Complete scale invariance follows from a similar argument. If we assume that the merger process begin only after the $\mathrm{L}_{0}$-size bubbles have reached their saturation velocity, then we can express the perturbation height $h(t)$ as the sum of the height at the saturation time $\mathrm{h}_{\text {sat }}$ plus the sum of the contributions of each generation:

$$
h(t)=h_{s a t}+\sum_{i=0}^{N} u_{i}^{a s y m} \tau_{i}=h_{s a t}+C_{2} L_{0}\left(2^{N+1}-1\right)
$$

Using (5) and (9), this can be written as

$$
h(t)-h_{\text {sat }}=C_{2}\left(2 L(t)-L_{0}\right)
$$

With the solution for L(t) in Eq. (11), this becomes

$$
h(t)-h_{s a t}=(\sqrt{2}-1)^{2} \frac{C^{2}}{C_{2}} g t^{2}+C(\sqrt{2}-1) \sqrt{L_{0} g} t .
$$


When condition (12) is satisfied, we are left with

$$
h(t)-h_{s a t}=\alpha(A) A g t^{2},
$$

where we have defined

$$
\alpha(A)=(\sqrt{2}-1)^{2} \frac{C^{2}}{C_{2} A} .
$$

Since $\mathrm{h}_{\text {sat }}$ is of order $\mathrm{L}_{0}$, it too can be neglected when (12) is satisfied if $\mathrm{C}_{2}$ is greater than or of order unity. This yields the familiar result

$$
h(t)=\alpha(A) A g t^{2}
$$

Thus at late times the initial scale $\mathrm{L}_{0}$ is not retained in the expression for the perturbation height. Together, Equations (13) and (19) show that $\mathrm{gt}^{2}$ emerges as the only remaining length scale in the problem. Since $\mathrm{L}(\mathrm{t})$ and $\mathrm{h}(\mathrm{t})$ have the same temporal scaling, the perturbation height is proportional to the wavelength, with

$$
\mathrm{h}(\mathrm{t}) / \mathrm{L}(\mathrm{t})=2 \mathrm{C}_{2} \text {, }
$$

and the system is said to be in the scale invariant regime. Note that asymptotic selfsimilarity follows directly from Eq. (15) and, for our general merger model [Eq. (7)], depends only on the assumption that merger occurs after the bubble has traversed a constant fraction of its diameter.

The mechanism for the loss of initial conditions is worth restating and can be easily visualized as follows. We rewrite Eq. (9) as follows to express the time at which the scale $\mathrm{L}$ is reached:

$$
t(L)=\frac{C_{2}}{C} \sqrt{\frac{L}{g}} \sum_{i=0}^{N} \frac{1}{2^{i / 2}}
$$

where

$$
\mathrm{N}=\log _{2}\left(\mathrm{~L} / \mathrm{L}_{0}\right)
$$


For large N, the sum in Eq. (21) is dominated by the first several terms, while the later terms are of less relative importance. The relative error of a partial sum from i equals zero to $\mathrm{n}<\mathrm{N}$ is given by Relative error $=\frac{2^{(N-n) / 2}-1}{\sqrt{2} 2^{N / 2}-1}$

Figure 2 is a plot of the $\mathrm{n}$ at which the sum is $90 \%$ converged, as a function of $\mathrm{N}$. Above $\mathrm{N}=10$, the the sum is dominated by the last seven terms. Thus as long as $\mathrm{N}$ is large, its precise value is not important in determining $\mathrm{t}(\mathrm{L})$. In other words, after many generations of bubble merger, the time to reach a given scale is only weakly dependent on the initial scale.

Before proceeding, there are a few points worth making. First of all, we note that we could have arrived at Eq. (19) by assuming from the beginning that the system eventually becomes self-similar. ${ }^{43}$ That is, we use Eq. (20) to replace L(t) in Eq. (3) with $h(t)$ and equate $u^{\text {asym }}$ with $\mathrm{dh} / \mathrm{dt}$. The solution of the resulting first-order ordinary differential equation is again Eq. (19), now with

$$
\alpha(\mathrm{A})=(1 / 4) \mathrm{C}^{2} /\left(\mathrm{C}_{2} \mathrm{~A}\right) \text {. }
$$

This result makes no direct assumption about the form of the merger rate and differs from Eq. (18) only in the numerical pre-factor, which is now 0.25 instead of $\approx 0.1716$. It is not surprising that the exact value of the pre-factor depends on whether the bubble diameter varies discreetly, as in deriving Eq. (18), or continuously, as for Eq. (24).

A second point is that for bubbles $\alpha(\mathrm{A})$ depends on A like $1 /(1+\mathrm{A})$, and therefore changes by only a factor of two over the entire range of possible Atwood numbers (neglecting in this instance the Atwood number dependence of our model's drag coefficient). This reasoning does not necessarily apply to spikes, though it must in the 
limit of vanishing Atwood number (in which case there is no difference between spikes and bubbles). As A approaches unity, $\alpha(A)$ for spikes must also approach unity so that spikes freefall with the acceleration of gravity. The model fails for spikes at A -> 1, where it predicts that $\alpha(\mathrm{A})$ increases without bound, because it is based on the assumption that the spikes have reached terminal velocity. Putting in reasonable values for the coefficients and assuming the above scaling for the merger time, we find $\alpha(\mathrm{A})=$ $0.036 /\left[\mathrm{C}_{2}(1+\mathrm{A})\right]$ for $2 \mathrm{D}$ bubbles and $\alpha(\mathrm{A})=0.097 /\left[\mathrm{C}_{2}(1+\mathrm{A})\right]$ for $3 \mathrm{D}$ bubbles [or $0.053 /\left(\mathrm{C}_{2}(1+\mathrm{A})\right)$ in $2 \mathrm{D}$ and $0.142 /\left(\mathrm{C}_{2}(1+\mathrm{A})\right)$ in $3 \mathrm{D}$ with the pre-factor in Eq. (24)]. For spikes, the expressions are the same if we make the substitution A ->-A. Despite the simplicity of our model and with $\mathrm{C}_{2} \approx 1$ as reported by Glimm and $\mathrm{Li}^{26}{ }^{26}$ these expressions agree to within about a factor of two with experiments and simulations. ${ }^{43}$

Finally, we consider what a more precise expression for the bubble merger time might look like. In a real system, merger events in general involve bubbles of different size that satisfy a merger criterion that depends on the dimension of the system. In $2 \mathrm{D}$, the bubble diameter is conserved $\left[\mathrm{L}_{\mathrm{i}+1}=\mathrm{L}_{\mathrm{i}}+\mathrm{L}_{\mathrm{j}} \equiv \mathrm{L}_{\mathrm{i}}+\kappa^{2} \mathrm{~L}_{\mathrm{i}}=\left(1+\kappa^{2}\right) \mathrm{L}_{\mathrm{i}} \equiv \mu \mathrm{L}_{\mathrm{i}}\right]$, while the bubble area is conserved in 3D $\left[\mathrm{L}_{\mathrm{i}+1}{ }^{2}=\mathrm{L}_{\mathrm{i}}{ }^{2}+\mathrm{L}_{\mathrm{j}}{ }^{2} \equiv \mathrm{L}_{\mathrm{i}}{ }^{2}+\kappa^{4} \mathrm{~L}_{\mathrm{i}}{ }^{2}=\left(1+\kappa^{4}\right) \mathrm{L}_{\mathrm{i}}^{2} \equiv \mu^{2} \mathrm{~L}_{\mathrm{i}}{ }^{2}{ }^{25} \mathrm{~A}\right.$ periodic array of identical bubbles is stable, so the merger time for two bubbles of equal size should actually be infinite. Finally, the expression for the merger time should be symmetric in $\mathrm{L}_{\mathrm{i}}$ and $\mathrm{L}_{\mathrm{j}}$. If we again adopt the convention of Sharp and Wheeler that bubble merger occurs when the height of the larger bubble above the top of the smaller bubble is a fraction $\mathrm{C}_{2}$ of the smaller bubble radius, ${ }^{25}$ then we find

$$
\tau\left(L_{i}\right)=\left(C_{2} / C\right) \sqrt{L_{i} / g} /(\kappa-1) .
$$

where $\mathrm{L}_{\mathrm{i}}$ is the smaller of $\mathrm{L}_{\mathrm{i}}$ and $\mathrm{L}_{\mathrm{j}}$ and 


$$
\kappa=\sqrt{L_{j} / L_{i}} .
$$

This result, which was also derived by Alon et al. ${ }^{36}$ for the Sharp-Wheeler bubble merger model, satisfies the requirements given above for a well-behaved merger time. The simple merger model discussed above has $\kappa=\sqrt{ } 2$, but all of its conclusions remain qualitatively unchanged for any other constant $\kappa$. Following the same procedure outlined above, we find that the transverse scale evolves according to

$$
L(t)=\left[\frac{\kappa-1}{C_{2}}\left(1-\frac{1}{\sqrt{\mu}}\right) \sqrt{\frac{|1-\eta|}{C_{D}} g t}+\sqrt{\frac{L_{0}}{\mu}}\right]^{2} .
$$

The bubble height growth is given by

$$
\begin{aligned}
h(t)= & h_{0}+\frac{C_{2}}{\kappa-1}\left(1-\frac{1}{\mu}\right)\left(L(t)-\frac{L_{0}}{\mu}\right) \\
& =\mathrm{h}_{0}+\frac{1-1 / \sqrt{\mu}}{1-1 / \mu} \frac{\kappa-1}{C_{2}}\left(1-\frac{1}{\sqrt{\mu}}\right) \frac{|1-\eta|}{C_{D}} g t^{2}+2 \frac{\kappa-1}{C_{2}}\left(1-\frac{1}{\sqrt{\mu}}\right) \sqrt{\frac{|1-\eta|}{C_{D}} \frac{L_{0}}{\mu}} g t \\
& \rightarrow \frac{1-1 / \sqrt{\mu}}{1-1 / \mu} \frac{\kappa-1}{C_{2}}\left(1-\frac{1}{\sqrt{\mu}}\right) \frac{|1-\eta|}{C_{D}} g t^{2}
\end{aligned}
$$

if $\mathrm{L}(\mathrm{t})$ varies discontinuously, resulting in the similarity parameter

$$
\frac{L(t)}{h(t)} \rightarrow \frac{\kappa-1}{C_{2}}\left(1-\frac{1}{\mu}\right)
$$

and by

$$
h(t)=\frac{1}{2} \frac{\kappa-1}{C_{2}}\left(1-\frac{1}{\sqrt{\mu}}\right) \frac{|1-\eta|}{C_{D}} g t^{2}
$$

if $\mathrm{L}(\mathrm{t})$ varies continuously, in which case we have

$$
\frac{L(t)}{h(t)} \rightarrow 2 \frac{\kappa-1}{C_{2}}\left(1-\frac{1}{\sqrt{\mu}}\right)
$$

In conclusion, we have used a very simple model of bubble merger to illustrate 
and motivate the general properties of late-time multimode RT instability evolution. Specifically, we have shown how the initial conditions are erased from both the transverse and parallel scales after times much longer than the first generation of bubble merger. This forces the system into a scale invariant regime in which the wavelength grows in proportion with the perturbation amplitude, and is based on the fact that smaller bubbles merge faster than larger bubbles. Loss of initial condition information is equivalent to the relative loss of importance of increasingly higher order terms in a converging series expansion. In addition, the model suggests why $\alpha(\mathrm{A})$ of the bubble might depend weakly, if at all, on the Atwood number.

\section{Blast-wave-driven interface motion}

The term blast wave is generally used to describe the fluid flow resulting from a strong explosion in a compressible medium, and the relevant scale-invariant solution to the Euler equations is discussed in the original works of Sedov ${ }^{44,45}$ and Taylor ${ }^{46}$ and in several other excellent references (see, for example, Landau and Lifshitz, ${ }^{47}$ Zel'dovich and Raizer, ${ }^{9}$ and Barenblatt $\left.{ }^{48}\right)$. Other than the most general overview of blast waves, it is important for our purposes to demonstrate RT instability due to the transmission of a blast wave through an interface, to determine the driving deceleration felt by the interface, and consider the effect of the large-scale fluid gradients on the developing instability. With this information in hand, we will extend the ideas of buoyancy-drag and statistical-mechanical merger models to the blast-wave-driven case. 
A blast wave results when a large amount of energy is released suddenly in a small volume within a compressible medium, as in an explosion. The expanding source acts as a piston, driving a shock wave into the surrounding material. Because of the impulsive nature of the drive, the shock strength decays as the front moves away from the center. As long as the pre-shock pressure can be neglected relative to the post-shock value (ie while the Mach number $M<<1$ ), the blast wave is described by a self-similar solution to the compressible Euler equations. In this idealized case, the only dimensional parameters in the problem are the energy released $(E)$ and the pre-shock density $\left(\rho_{0}\right)$ in units of mass per (length) ${ }^{d}$ where $d$ is the dimension of the blast wave. Blast waves can be spherical $(d=3)$ as in the case of supernovae or other unconfined explosions, cylindrical $(d=2)$, or planar $(d=1)$. The term "planar blast wave" is often used to describe the flow in impulsively-driven shock tubes, including the millimeter-scale laser-driven tubes used for laboratory astrophysics experiments designed to study compressible mixing in supernovae. ${ }^{16-24}$ The motion of a gas under the action of an impulsive load is similar to, but distinct from, the solution for a planar blast wave. Whereas the position of the planar blast front scales like $\mathrm{t}^{2 / 3}$ independent of material compressibility (see below), the result in the impulsive load problem is $\mathrm{t}^{\alpha}$ where $\alpha=0.5$ at $\gamma=1$ and approaches $2 / 3$ as $\gamma$ tends to infinity. ${ }^{9}$ These differences can be approximately accounted for by applying the generalized model discussed below, which allows for arbitrary acceleration profile and velocity gradient.

The d-dimensional "radius" of the shock front must then be proportional to $\left(\mathrm{Et}^{2} / \rho_{0}\right)^{1 /(\mathrm{d}+2)}$, the only length scale that can be formed from these parameters and time. We define the constant of proportionality $\xi_{0}$ so that the shock displacement $r_{\mathrm{s}}$ is given by 


$$
r_{s}=\xi_{0}\left(\frac{E t^{2}}{\rho_{0}}\right)^{\frac{1}{d+2}}
$$

This expression is differentiated to determine the shock's velocity and deceleration as

$$
\begin{aligned}
& v_{s}=\frac{2}{d+2} \xi_{0}\left(\frac{E t^{2}}{\rho_{0}}\right)^{\frac{1}{d+2}} \frac{1}{t}=\frac{2}{d+2} \frac{r_{s}}{t} \\
& g_{s}=\frac{2}{d+2} \frac{d}{d+2} \frac{r_{s}}{t^{2}}=\frac{d}{d+2} \frac{v_{s}}{t}
\end{aligned}
$$

The similarity variable

$$
\xi=r\left(\frac{\rho_{0}}{E t^{2}}\right)^{\frac{1}{d+2}}=\xi_{0} \frac{r}{r_{s}}
$$

varies from zero at the center to $\xi_{0}$ at the shock. Except for a time-dependent scale factor, the fluid variables must depend only on the similarity variable. The scale factors are determined by the strong-shock limit of the Rankine-Hugoniot relations, which for a polytropic equation of state require that the post-shock density, pressure, and fluid velocity are given by

$$
\begin{aligned}
& \rho_{s}=\frac{\gamma+1}{\gamma-1} \rho_{0} \\
& p_{s}=\frac{2}{\gamma+1} \rho_{0} v_{s}^{2} . \\
& u_{s}=\frac{2}{\gamma+1} v_{s}
\end{aligned}
$$

When the functions

$$
\begin{aligned}
& \rho(r, t)=\rho(\xi)=\rho_{s} \phi(\xi) \\
& p(r, t)=\rho(\xi, t)=p_{s}(t) \emptyset(\xi) \\
& u(r, t)=u(\xi, t)=u_{s}(t) \downarrow f(\xi)
\end{aligned}
$$

are inserted into the Euler equations, they reduce to a set of ordinary differential equations for the dimensionless functions $\oint(\xi), \not(\xi)$, and $\mathscr{f}(\xi)$. These equations can be solved numerically or analytically, and a typical solution is shown in Fig. 3. Because the 
pressure falls of monotonically behind the shock front, an interface between two fluids is RT-unstable when it transmits a blast wave from the more dense to the less dense material.

The velocity of a lagrangian fluid element behind the shock front is determined by

$$
\begin{aligned}
\frac{d u(\xi, t)}{d t} & =\frac{\partial u(\xi, t)}{\partial t}+\frac{\partial \xi}{\partial t} \frac{\partial u(\xi, t)}{\partial \xi}=y \frac{\partial u_{s}}{\partial t}+u_{s} \frac{d \xi}{d t} \frac{\partial f}{\partial \xi} \\
& =-\frac{2}{\gamma+1} g_{s}(t) y(\xi)\left[1+\frac{2}{d} \frac{\xi}{v(\xi)} \frac{\partial f(\xi)}{\partial \xi}\left(1-\frac{2 \xi_{0}}{\gamma+1} \frac{f(\xi)}{\xi}\right)\right],
\end{aligned}
$$

where the first term describes the decrease in time of the fluid velocity scale factor (the post-shock fluid velocity) and the second term describes the motion of the fluid element along the similarity solution. In obtaining this result, we have used the evolution equation of the similarity variable, which is given by

$$
\begin{aligned}
\frac{d \xi(r, t)}{d t} & =\frac{\partial \xi(r, t)}{\partial t}+u \frac{\partial \xi(r, t)}{\partial r} \\
& =-\frac{2}{d+2} \frac{\xi}{t}\left(1-\frac{2 \xi_{0}}{\gamma+1} \frac{y(\xi)}{\xi}\right)
\end{aligned}
$$

Given that the solution to the scale-invariant function $f(\xi)$ is known, Eq. (38) can be solved and its solution inserted into Eq. (37). This then gives the interface acceleration history $g_{i}(t)=d u\left(\xi_{i}(t), t\right) / d t$ that drives the RT instability.

The full analytic solution to the blast-wave problem with a polytropic EOS is rather cumbersome, but the asymptotic $(\xi \rightarrow 0)$ form of $\downarrow f(\xi)$ is quite simple and given by

$$
\mathscr{f}(\xi)=\frac{\gamma+1}{2 \gamma} \frac{\xi}{\xi_{0}} \Rightarrow u(r, t)=\frac{2}{d+2} \frac{1}{\gamma} \frac{r}{t}
$$

In fact, this asymptotic solution is generally a good approximation to the full solution everywhere except just behind the shock front, where it under-predicts the velocity gradient. The boundary condition at the shock front is 


$$
\mathscr{f}\left(\xi_{0}\right)=1 \Rightarrow u\left(r_{s}, t\right)=\frac{2 \gamma}{\gamma+1} \frac{2}{d+2} \frac{1}{\gamma} \frac{r_{s}}{t}
$$

so the range of validity of the asymptotic solution is extended for more compressible $(\gamma$ approaching unity) materials.

Equation (39) shows that, away from the shock front, the flow is characterized by a linear velocity gradient that decays in time, as in a centered rarefaction wave. Thus a blast wave can be thought of as a shock wave plus a rarefaction wave.

If we insert the asymptotic solution Eq. (39) into Eq. (38) and integrate, we find that

$$
\xi_{i}(t)=\xi_{0}\left(\frac{t_{0}}{t}\right)^{\frac{2}{d+2} \frac{\gamma-1}{\gamma}}
$$

where $t_{0}=\sqrt{\rho / E}\left(r_{0} / \xi_{0}\right)^{(d+2) / 2}$ is the time at which the shock reaches the interface. The corresponding interface deceleration is then

$$
g_{i}(t)=\frac{r_{0}}{t_{0}{ }^{2}} \frac{2}{\gamma(d+2)}\left[1-\frac{2}{\gamma(d+2)}\right]\left(\frac{t_{0}}{t}\right)^{2\left[1-\frac{1}{\gamma(d+2)}\right]},
$$

which can be integrated to obtain the interface velocity and position:

$$
\begin{aligned}
& u_{i}(t)=\frac{r_{0}}{t_{0}} \frac{2}{\gamma(d+2)}\left(\frac{t_{0}}{t}\right)^{1-\frac{2}{\gamma(d+2)}} \\
& r_{i}(t)=r_{0}\left(\frac{t}{t_{0}}\right)^{\frac{2}{\gamma(d+2)}} \cdot
\end{aligned}
$$

The position, velocity, and deceleration of the shock are recovered by setting $\gamma \rightarrow 1$ in Eqs. (42)-(44). This means that, for an infinitely compressible medium, the interface remains at the shock front at all times. As is the case of a steady shock, a more general $\gamma$ describes how quickly the shock pulls away from the interface. 


\section{Buoyancy-drag model for blast-wave-driven case}

With expressions for the interface deceleration and velocity gradient in hand (Eq.'s 39 and 42), we now proceed to generalize the simple buoyancy-drag and merger models presented in Section II model to the case of an interface driven by a strong blast wave. In particular, we consider how compressibility and the time-dependence of the driving deceleration change the main conclusions of Section II. This extension entails three main complications.

First of all, the blast-wave-driven interface is unstable to the Richtmyer-Meshkov (RM) instability ${ }^{49.50}$ in addition to RT. The RM instability results when a shock crosses a material interface, whether from light to heavy or heavy to light. The shock deposits vorticity via the baroclinic term in the vorticity equation, and the evolution of the deposited vorticity field results in perturbation growth. The simplest model of RM growth is the impulsive model, originally presented by Richtmyer, ${ }^{49}$ which treats the action of the shock as a delta-function acceleration. The impulsive model predicts that the instability grows linearly in time while the perturbation amplitude is small compared to its wavelength.

When both RM and RT are present, they do not necessarily add linearly, and there is to our knowledge no general (non-phenomenological) model that includes the effects of both. Simulations of blast-wave-driven laser experiments suggest that, for strong blast waves, RM dominates the instability growth very early on while RT dominates at later 
times. ${ }^{24}$ Single-mode simulations were well modeled by a buoyancy-drag model in which $\mathrm{RM}$ is approximately accounted for by simply setting the initial spike and bubble velocities equal to those predicted by the impulsive model. In what follows, we assume that the main effect of the RM component is to decrease the time required for the instability to reach the nonlinear state where the buoyancy-drag model is applicable, and make no additional accounting for RM effects.

The second complication is the time-dependence of the driving acceleration. For an interface driven by a blast wave, the deceleration is greatest just after passage of the shock front and subsequently decays in time. With a time-dependent acceleration, the number of possible length scales that can be formed in the problem independent of the perturbation scales is infinite $\left[\left(\int \mathrm{dt}^{\mathrm{n}} \mathrm{g}(\mathrm{t})^{\mathrm{n} / 2}\right)^{2 / \mathrm{n}}\right.$ for all positive integer $\left.\mathrm{n}\right]$. In the classical case, these collapse into the single scale $\mathrm{gt}^{2}$. If we allow $\mathrm{g}$ in Eq. (3) to vary in time and still assume self-similarity, then we find that the mix width growth should scale like $\mathrm{e}^{34,43}$

$$
\mathrm{h} \sim\left[\int \mathrm{dt} \mathrm{g}^{1 / 2}\right]^{2}
$$

Generalizing the bubble-merger model presented for the classical case [in particular Eq. (24)], we find that the merger-time is given by

$$
(\kappa-1) \int_{t_{i}}^{t_{i}+\tau_{i}} u^{a s y m}\left(t^{\prime}\right) d t^{\prime}=(\kappa-1) C \sqrt{L_{i}} \int_{t_{i}}^{t_{i}+\tau_{i}} \sqrt{g\left(t^{\prime}\right)} d t^{\prime}=C_{2} L_{i} .
$$

in the time-dependent acceleration case. Strictly speaking, $g(t)$ in Eq. (46) is the acceleration at the position of the bubble tip. In compressible systems, this is not necessarily the acceleration at the unperturbed-interface location. For the time being, we will nevertheless use the interface acceleration $\mathrm{g}_{\mathrm{i}}(\mathrm{t})$ as the drive for bubble growth, effectively assuming that the acceleration varies little over the bubble height. Summing 
both sides of (46) from $\mathrm{i}=1$ to $\mathrm{N}$ as before, we find that the transverse scale grows according to

$$
L(t)=\left[\frac{\kappa-1}{C_{2}}\left(1-\frac{1}{\sqrt{\mu}}\right) \int_{t_{0}}^{t} \sqrt{\frac{|1-\eta|}{C_{D}} g\left(t^{\prime}\right)} d t^{\prime}+\sqrt{\frac{L_{0}}{\mu}}\right]^{2},
$$

and that the late-time bubble amplitude height is given by

$$
h(t) \rightarrow \frac{\kappa-1}{C_{2}}\left(1-\frac{1}{\sqrt{\mu}}\right) \int_{t_{0}}^{t} \int_{t_{0}}^{t^{\prime}} d t^{\prime} d t^{\prime \prime} \sqrt{\frac{|1-\eta|}{C_{D}} g\left(t^{\prime}\right)} \sqrt{\frac{|1-\eta|}{C_{D}} g\left(t^{\prime \prime}\right)} .
$$

The ratio of (47) to (48) gives the same similarity parameter as in the constant $g$ case:

$$
\frac{L(t)}{h(t)} \rightarrow 2 \frac{\kappa-1}{C_{2}}\left(1-\frac{1}{\sqrt{\mu}}\right)
$$

since $\int_{t_{0}}^{t} \int_{t_{0}}^{t^{\prime}} d t^{\prime} d t^{\prime \prime} \sqrt{\frac{|1-\eta|}{C_{D}} g\left(t^{\prime}\right)} \sqrt{\frac{1-\eta \mid}{C_{D}} g\left(t^{\prime \prime}\right)}=\frac{1}{2}\left[\int_{t_{0}}^{t} d t^{\prime} \sqrt{\frac{|1-\eta|}{C_{D}} g\left(t^{\prime}\right)}\right]^{2}$. Again, we recover the $\left[\int d t g^{1 / 2}\right]^{2}$ scaling for both $\mathrm{L}(\mathrm{t})$ and $\mathrm{h}(\mathrm{t})$ after several merger generations. Experimental data obtained on a rocket-rig apparatus by $\operatorname{Read}^{34}$ and Dimonte et al. ${ }^{43}$ agree with this scaling for both rising and falling accelerations. This suggests that time dependence alone does not invalidate the ideas of scale invariance and loss of initial conditions.

However, it is important to note that Eq. (42) for the interface deceleration, though non-zero at all times, is only valid while the Mach number of the shock is high enough that we can neglect the pre-shock pressure $\left(M^{2}>>1 \Rightarrow M \ngtr 3\right)$. For a real blast wave, the Mach number eventually approaches unity and the interface acceleration goes to zero. Thus the interface is driven for a finite time that we can approximate as

$$
\frac{t_{m}}{t_{0}}=\left(\frac{2}{d+2} \frac{r_{0}}{c t_{0}}\right)^{(d+2) / d}=\left(\frac{2}{d+2} M_{0}^{a v e}\right)^{(d+2) / d}
$$

or 


$$
\frac{t_{m}}{t_{0}}=\left(\frac{2}{3(d+2)} \frac{r_{0}}{c t_{0}}\right)^{(d+2) / d}=\left(\frac{2}{3(d+2)} M_{0}^{a v e}\right)^{(d+2) / d}
$$

which is the time at which Eq. (32) predicts that the shock speed is equal to the pre-shock sound speed c (Eq. 50a and Fig. 4a) or three times the sound speed (Eq. 50b and Fig. 4b). In this expression, $\mathrm{M}_{0}$ ave is the average Mach number up to $\mathrm{t}_{0}$. A significant change in the shock speed due to transmission through the interface can be approximately accounted for by rescaling $\mathrm{t}_{0}$ on the right-hand side of Eq. (50). If the bubble amplitude grows to the point that it is not small compared to the interface coordinate $r_{i}$, then the drive at the bubble tip falls below the interface deceleration and the value of $t_{m}$ will consequently be somewhat reduced.

For blast-wave-driven systems, loss of memory of initial conditions can occur only if the time-dependent term in Eq. (47) becomes large compared to the constant term, which includes the initial transverse scale:

$$
\frac{\kappa-1}{C_{2}}\left(1-\frac{1}{\sqrt{\mu}}\right) \int_{t_{0}}^{t} \sqrt{\frac{|1-\eta|}{C_{D}} g\left(t^{\prime}\right)} d t^{\prime}>>\sqrt{\frac{L_{0}}{\mu}} .
$$

Because of the drive decay, this condition must be satisfied in a time $t<\mathrm{t}_{\mathrm{m}}$.

The third complication is the violation of the assumption of incompressibility. For a blast wave propagating through a single material that is initially homogeneous and isotropic, the density falls off monotonically as the material decompresses in the rarefaction behind the shock front. Because of the density gradient, spikes and bubbles experience decreasing Atwood numbers as their amplitudes increase. ${ }^{40}$ This is a relatively small effect for high Atwood number systems in the nonlinear regime, and is not accounted for in our model. A more significant effect results from the velocity gradient associated with the density gradient. In the self-similar regime (while the blast wave 
Mach number is large), the post-shock fluid velocity is approximately proportional to $\mathrm{r} / \mathrm{t}$ (the $\xi \rightarrow 0$ asymptotic result where $\xi$ is the similarity variable) except for just behind the shock front. Decompression provides another source of perturbation growth in addition to the RT and RM instabilities. We can account for the post-shock material decompression by adding the a term $\beta_{b, s} h_{b, s} / t$, where $h_{b, s}$ is the bubble (spike) amplitude, to Eq. (3) for the terminal velocity of the bubble or spike:

$$
\frac{d h_{b, s}(t)}{d t}=\sqrt{\frac{\left|1-\eta_{b, s}\right|}{C_{D}} \lambda g(t)}+\beta_{b, s} \frac{h_{b, s}}{t}
$$

where again $\eta_{\mathrm{b}, \mathrm{s}}=\rho_{\mathrm{b}, \mathrm{s}} / \rho_{\mathrm{s}, \mathrm{b}}$. The terminal velocity now depends explicitly on time and on the bubble or spike height in addition to the transverse scale and time-dependent acceleration. The coefficient $\beta$ is given in Eq. (39) as $\frac{2}{\gamma(d+2)}$ for the case of an ideal blast wave away from the shock front. Equation (52) is valid, however, for any RT-unstable system in a velocity gradient proportional to $r / t$ and interface position that scales like $\mathrm{t}^{\mathrm{\beta}}$. This allows for fits in systems driven by non-ideal blast waves and when $\beta$ is different in each of the spike and bubble regions. This is the only accounting we make within the model for any deviations from the single-material blast-wave solution resulting from shock transmission through the interface. Again, the situation is complicated if we account for the fact that the acceleration at the spike and bubble tips is not that same as at the unperturbed interface position. It follows from Eqs. (31), (42), and (44) that $g_{b, s}(t)=g_{i}(t)\left[1 \mp h_{b, s}(t) / r_{i}(t)\right]$, where the minus (plus) sign is for the bubble (spike). The approximation $g_{b, s}(t) \approx g_{i}(t)$, which we will always make, is valid when the perturbation amplitude is small compared to the interface radius. 
Equation (52) can be integrated to yield the perturbation amplitude history for a single mode driven by a strong planar blast wave:

$$
h(t)=h\left(t_{0}\right)\left(\frac{t}{t_{0}}\right)^{\frac{2}{\gamma(d+2)}}+2 r_{0} \sqrt{\frac{\lambda}{r_{0}} \frac{|1-\eta|}{C_{D}}\left(\frac{\gamma(d+2)}{2}-1\right)}\left(\frac{t}{t_{0}}\right)^{\frac{1}{\gamma(d+2)}}\left[\left(\frac{t}{t_{0}}\right)^{\frac{1}{\gamma(d+2)}}-1\right] . \quad \mathrm{d}=1
$$

The effect of stretching due to material decompression is present both in the first term, resulting in stretching of the initial perturbation, and in the second term, giving enhanced stretching as the perturbation grows in amplitude. If we remove the time-dependent factor in the first term and the first time-dependent factor in the second term, we recover the result for a perturbation driven by a blast-wave acceleration but without decompression. In the absence of decompression, the relative importance of the first term, which includes the initial amplitude of the perturbation, tends to zero as t tends to infinity. Significantly, the relative importance of the initial amplitude does not tend to zero when decompression is present. Asymptotically, the ratio of the first term in Eq. 53 to the second approaches a constant value of $\frac{h_{0}}{\sqrt{r_{0} \lambda}} \sqrt{\frac{C_{D}}{6 \gamma|1-\eta|} \frac{1}{1-\frac{2}{3 \gamma}}} \sim \frac{h_{0}}{\sqrt{r_{0} \lambda}}\left\{1, \eta^{-1 / 2}\right\}_{b, s}$, where the first factor is for bubbles and the second for spikes, for a planar blast wave. This will typically be much less than one even for bubbles, but could be significant for initially nonlinear perturbations on an interface initially located no more than a few wavelengths away from the center.

For interfaces driven by multidimensional blast waves $(\mathrm{d}>1)$, the wavelength grows in time due to divergence according to

$$
\lambda(t)=2 \pi r_{i}(t) / m=\lambda_{0} r_{i}(t) / r_{0},
$$


where $m=2 \pi r_{0} / \lambda_{0}$ is the perturbation mode number, and Eq. (53) is no longer valid. Instead, using Eq. (54) in Eq. (52), we find

$$
h(t)=h\left(t_{0}\right)\left(\frac{t}{t_{0}}\right)^{\frac{2}{\gamma(d+2)}}+\frac{2 r_{0}}{\gamma(d+2)} \sqrt{\frac{\lambda_{0}}{r_{0}} \frac{1-\eta}{C_{D}}\left(\frac{\gamma(d+2)}{2}-1\right)}\left(\frac{t}{t_{0}}\right)^{\frac{2}{\gamma(d+2)}} \ln \left(\frac{t}{t_{0}}\right) . \quad \mathrm{d}>1
$$

Unlike the planar case, the relative contribution of the initial amplitude in diverging systems tends to zero as late times (though only logarithmically). The continually increasing wavelength gives a continually increasing growth rate for a given acceleration, but the acceleration in higher dimensions falls off much faster than in 1D. The net result is that at late times the instability grows faster in $1 \mathrm{D}$ than in higher dimensions.

Rather than restricting ourselves to ideal blast waves, we also consider the more general case where we only require that the instability develop in a linear velocity gradient given by $\Delta \mathrm{u}=\beta \Delta \mathrm{r} / \mathrm{t}$, and that the interface trajectory follow

$$
r_{i}(t)=r_{0}\left(\frac{t}{t_{0}}\right)^{\beta}
$$

The interface is driven by an arbitrary acceleration $\mathrm{g}(\mathrm{t})$, and the evolution of a single mode is given by

$$
h(t)=h\left(t_{0}\right)\left(\frac{t}{t_{0}}\right)^{\beta}+r_{0} \sqrt{\frac{|1-\eta|}{C_{D}}}\left(\frac{t}{t_{0}}\right)^{\beta} \int_{t_{0}}^{t}\left(\frac{t^{\prime}}{t_{0}}\right)^{-\beta} \sqrt{\frac{\lambda\left(t^{\prime}\right)}{r_{0}} \frac{g\left(t^{\prime}\right) t_{0}^{2}}{r_{0}}} \frac{d t^{\prime}}{t_{0}},
$$

where the wavelength is time-dependence only in diverging systems $(d>1)$.

This more general version of the model can be applied to laser-driven RT experiments in which $\mathrm{a} \sim 1 \mathrm{~ns}$ pulse from a high-powered laser is used to drive a decaying shock into a millimeter-scale beryllium shock tube. Though it resembles (and is often referred to as) a planar blast wave, the drive deviates somewhat from the selfsimilar Taylor-Sedov solution discussed above. Figure 5 shows a comparison of Eq. (57) 
with an $\mathrm{A}=0.7$ Raptor $^{51}$ simulation of a proposed experiment in which a $1 \mathrm{~ns} 25 \mathrm{~kJ}$ drive is applied to a single mode perturbation with $50 \mu \mathrm{m}$ wavelength and $2.5 \mu \mathrm{m}$ initial amplitude (The simulations and related experiments are discussed in detail elsewhere ${ }^{16-}$ ${ }^{24,52}$ ). The model curve (dashed line) with $\beta=0.51$ agrees very well with the bubble amplitude history predicted by the simulation (solid line). A value for $\beta$ can be obtained from the simulation either by measuring the velocity gradient, which gives $\beta \approx 0.61$ or plotting the interface trajectory [using Eq. (56)], which gives $\beta \approx 0.42$. The average of these two gives the best agreement between model and simulation. Because the model assumes that spikes and bubbles instantaneously reach their terminal velocities, it does not accurately describe the spike growth at high Atwood number. In such cases, one would have to numerically solve the buoyancy-drag differential equation [Eq. (2a)] for the spike velocity relative to the flow at the spike-tip position, adding at each time step the decompression velocity $\beta h_{s}(t) / t$ to find the velocity relative to the unperturbed interface. This is not necessary, however, in order to qualitatively capture the bubblefront evolution and associated inverse cascade to larger transverse scales.

\section{Merger model for blast-wave-driven case}

We now consider how the merger model presented in Section II is altered in the blast-wave-driven case. Rather than assume that the basic tenets of the model are the same regardless of the relative sizes of the merging bubbles, we again treat the more general case where a bubble of diameter L mergers with a larger bubble with diameter 
$\kappa^{2} \mathrm{~L}(\kappa>1)$. For a merger event that begins at time $t_{i}$, the difference in height between the two bubbles reaches a value of $\mathrm{C}_{2} \mathrm{~L}$ at time $\mathrm{t}_{\mathrm{i}+1}=\mathrm{t}_{\mathrm{i}}+\tau_{\mathrm{i}}$, where $\tau$ is the merger time.

Equation (57) for motion of a single bubble is applied to two bubbles of diameter L and $\kappa^{2} \mathrm{~L}$, and the merger time is determined from their difference:

$$
\begin{aligned}
& C_{2} \sqrt{L_{i}}\left\{\left(\frac{t_{i+1}}{t_{0}}\right)^{-\beta},\left(\frac{t_{i}}{t_{0}}\right)^{-\beta / 2}\right\}_{d=1, d>1}=\frac{\Delta h_{i}}{\sqrt{L_{i}}}\left\{\left(\frac{t_{i}}{t_{0}}\right)^{-\beta},\left(\frac{t_{i}}{t_{0}}\right)^{-\beta / 2}\right\}+ \\
& \sigma_{0}^{-1} \sqrt{r_{0}} \int_{t_{i}}^{t_{i+1}}\left(\frac{t}{t_{0}}\right)^{-\beta} \sqrt{\frac{t_{0}^{2} g(t)}{r_{0}}}\left\{1,\left(\frac{t}{t_{0}}\right)^{-\beta / 2}\right\} \frac{d t}{t_{0}}
\end{aligned}
$$

In (58), we have defined the parameter $\sigma_{0}^{-1} \equiv(\kappa-1) \sqrt{|1-\eta| / C_{D}}$ and $\Delta \mathrm{h}_{\mathrm{i}}=\mathrm{h}_{\mathrm{kL}}\left(\mathrm{t}_{\mathrm{i}}\right)-\mathrm{h}_{\mathrm{L}}\left(\mathrm{t}_{\mathrm{i}}\right)$ is the separation between the two bubbles at $t_{\mathrm{i}}$. The distinction between the $\mathrm{d}=1$ and $\mathrm{d}>1$ is important because, in the divergent case $(d>1)$, we require that the difference in height between the two bubbles reach $\mathrm{C}_{2} \mathrm{~L}\left(\mathrm{t}_{\mathrm{i}+1}\right)=\mathrm{C}_{2} \mathrm{~L}\left(\mathrm{t}_{\mathrm{i}}\right)\left(t_{i+1} / t_{i}\right)^{\beta}$ before merger.

Recalling that $\mu \equiv 1+\kappa^{2}$ for $2 \mathrm{D}$ perturbations and $\mu \equiv \sqrt{1+\kappa^{4}}$ for $3 \mathrm{D}$ perturbations, we find that

$$
L_{i}=\left\{\mu^{i} L_{0}, \frac{2 \pi r_{i}\left(t_{i}\right)}{m_{i}}\right\}_{d=1, d>1}=\left\{\mu^{i} L_{0}, 2 \pi r_{0}\left(t_{i}\right)\left(\frac{t_{i}}{t_{0}}\right)^{\beta} \frac{\mu^{i}}{m_{0}}\right\}=\mu^{i} L_{0}\left\{1,\left(\frac{t_{i}}{t_{0}}\right)^{\beta}\right\} .
$$

Inserting (59) into (58), we find

$$
\begin{aligned}
\mu^{i / 2} \sqrt{L_{0}}\left\{\left(\frac{t_{i+1}}{t_{0}}\right)^{-\beta}, 1\right\}_{d=1, d>1}= & \frac{\Delta h_{i}}{C_{2} \mu^{i / 2} \sqrt{L_{0}}}\left\{\left(\frac{t_{i}}{t_{0}}\right)^{-\beta},\left(\frac{t_{i}}{t_{0}}\right)^{\beta / 2}\right\}+ \\
& \frac{\sqrt{r_{0}}}{C_{2} \sigma_{0}} \int_{t_{i}}^{t_{i+1}}\left(\frac{t}{t_{0}}\right)^{-\beta} \sqrt{\frac{t_{0}^{2} g(t)}{r_{0}}}\left\{1,\left(\frac{t}{t_{0}}\right)^{-\beta / 2}\right\} \frac{d t}{t_{0}}
\end{aligned}
$$

As in the incompressible case, we wish to sum both sides in i to find an implicit relation for $\mathrm{L}_{\mathrm{N}}=\mathrm{L}\left(\mathrm{t}_{\mathrm{N}}\right)=\mathrm{L}_{0} \mu^{\mathrm{N}} \equiv \mathrm{L}(\mathrm{t})$. Carrying out the sum, we find 


$$
\begin{aligned}
\left\{\sqrt{L_{0}} \sum_{i=0}^{N} \mu^{i / 2}\left(\frac{t_{i+1}}{t_{0}}\right)^{-\beta}, \frac{\sqrt{2 \pi r_{0} \mu / m_{N}}-\sqrt{L_{0}}}{\sqrt{\mu}-1}\right\}_{d=1, d>1}= & \sum_{i=0}^{N} \frac{\Delta h_{i}}{C_{2} \mu^{i / 2} \sqrt{L_{0}}}\left\{\left(\frac{t_{i}}{t_{0}}\right)^{-\beta},\left(\frac{t_{i}}{t_{0}}\right)^{\beta / 2}\right\}+ \\
& \frac{\sqrt{r_{0}}}{C_{2} \sigma_{0}} \int_{t_{0}}^{t_{N}}\left(\frac{t}{t_{0}}\right)^{-\beta} \sqrt{\frac{t_{0}^{2} g(t)}{r_{0}}}\left\{1,\left(\frac{t}{t_{0}}\right)^{-\beta / 2}\right\} \frac{d t}{t_{0}}
\end{aligned}
$$

where the perturbation mode number $m_{N}=m_{0} / \mu^{N}=2 \pi r_{0} /\left(\mu^{N} L_{0}\right)$. An important consequence follows from the fact that the sum on the left-hand side of Eq. (61) depends on the drive in the planar case but is independent of the drive in divergent systems. The second term on the right-hand side, which dominates at late times, is independent of the initial conditions. If the left-hand side reduces to a function of $\mathrm{L}(\mathrm{t})$ that is has no explicit dependence on $\mathrm{L}_{0}$ in the limit $\mathrm{L}(\mathrm{t}) / \mathrm{L}_{0}->$ infinity, then loss of memory of initial conditions is possible. For $d=1$, this is not true in general for arbitrary $g(t)$, and can only be true for special choices of the merger time such as $\frac{t_{i+1}}{t_{0}}=\left(\frac{L_{0} \mu^{i}}{r_{0}}\right)^{p}=\left(\frac{L_{i}}{r_{0}}\right)^{p}$ for arbitrary p. For planar blast waves, we will show that this condition is approximately met only within a small region of the parameter space.

For diverging systems, on the other hand, we have

$$
m(t)=\left[\frac{1}{C_{2} \sigma_{0}} \frac{\sqrt{\mu}-1}{\sqrt{2 \pi \mu}} \int_{t_{0}}^{t}\left(\frac{t}{t_{0}}\right)^{-\beta / 2} \sqrt{\frac{t_{0}^{2} g(t)}{r_{0}}} \frac{d t}{t_{0}}+\sqrt{\frac{L_{0}}{2 \pi r_{0} \mu}}\right]^{-2} . \quad \mathrm{d}>1
$$

At late times, the mode number is independent of the initial conditions, as is the transverse scale

$$
L(t)=\left[\frac{\kappa-1}{C_{2} \sigma_{0}}\left(1-\frac{1}{\sqrt{\mu}}\right) \int_{t_{0}}^{t}\left(\frac{t}{t_{0}}\right)^{-\beta / 2} \sqrt{\frac{t_{0}^{2} g(t)}{r_{0}}} \frac{d t}{t_{0}}+\sqrt{\frac{L_{0}}{r_{0} \mu}}\right]^{2}\left(\frac{t}{t_{0}}\right)^{\beta} \cdot \mathrm{d}>1
$$


Inserting the time-dependent transverse scale into Eq. (57), we find that the mix width grows according to

$$
d>1
$$

$$
\begin{aligned}
& \frac{h(t)}{r_{0}}=\left\{\begin{array}{l}
\frac{h_{0}}{r_{0}}+\frac{1}{\sqrt{\mu}} \sqrt{\frac{L_{0}}{r_{0}}} \int_{t_{0}}^{t} d t^{\prime}\left(\frac{t^{\prime}}{t_{0}}\right)^{-\beta / 2} \sqrt{\frac{|1-\eta|}{C_{D}} \frac{t_{0}^{2} g\left(t^{\prime}\right)}{r_{0}}}+ \\
\frac{\kappa-1}{C_{2}}\left(1-\frac{1}{\sqrt{\mu}}\right) \int_{t_{0}}^{t} \int_{t_{0}}^{t^{\prime}} d t^{\prime} d t^{\prime \prime}\left(\frac{t^{\prime}}{t_{0}}\right)^{-\beta / 2} \sqrt{\frac{|1-\eta|}{C_{D}} \frac{t_{0}^{2} g\left(t^{\prime}\right)}{r_{0}}}\left(\frac{t^{\prime \prime}}{t_{0}}\right)^{-\beta / 2} \sqrt{\frac{|1-\eta|}{C_{D}} \frac{t_{0}^{2} g\left(t^{\prime \prime}\right)}{r_{0}}}
\end{array}\right\}\left(\frac{t}{t_{0}}\right)^{\beta} \\
& =\left\{\frac{h_{0}}{r_{0}}+\frac{1}{\sqrt{\mu}} \sqrt{\frac{L_{0}}{r_{0}}} \int_{t_{0}}^{t} d t^{\prime}\left(\frac{t^{\prime}}{t_{0}}\right)^{-\beta / 2} \sqrt{\frac{1-\eta}{C_{D}} \frac{t_{0}^{2} g\left(t^{\prime}\right)}{r_{0}}}+\frac{1}{2} \frac{\kappa-1}{C_{2}}\left(1-\frac{1}{\sqrt{\mu}}\right)\left[\int_{t_{0}}^{t} d t^{\prime}\left(\frac{t^{\prime}}{t_{0}}\right)^{-\beta / 2} \sqrt{\frac{|1-\eta|}{C_{D}} \frac{t_{0}^{2} g\left(t^{\prime}\right)}{r_{0}}}\right]^{2}\right\}\left(\frac{t}{t_{0}}\right)^{\beta}
\end{aligned}
$$

In this general form [as in Eq. (48)], the perturbative nature of the model is apparent. The last term on the right-hand side, which describes the interaction between two bubbles, is the first-order nonlinear term in a multi-bubble interaction expansion. In truncating the series after the first nonlinear term, we are assuming that interactions between three or more bubbles are insignificant compared to pair interactions. Note also that the initial amplitude appears only in the first term on the right-hand side and the initial transverse scale appears only in the second term on the right-hand side. The third term, which dominates at late times (as long as $\mathrm{g}(\mathrm{t})$ falls off slower than $1 / \mathrm{t}^{2}$ ) is again independent of the initial conditions, and we again find an asymptotically-self-similar state with the same constant ratio of transverse to parallel scales as in the incompressible case:

$$
\frac{L(t)}{h(t)} \rightarrow 2 \frac{\kappa-1}{C_{2}}\left(1-\frac{1}{\sqrt{\mu}}\right) . \quad \mathrm{d}>1
$$

In fact, Eqs. (63) and (64) are identical to Eqs. (47) and (48) for the incompressible case except for the stretching factors $\left(\mathrm{t} / \mathrm{t}_{0}\right)^{\beta}$ and $\left(\mathrm{t} / \mathrm{t}_{0}\right)^{-\beta / 2}$ in the integrals. Because of the $\left(\mathrm{t} / \mathrm{t}_{0}\right)^{-\beta / 2}$ factors, the time required for loss of memory of initial conditions is longer for systems in a parallel velocity gradient then it would be in incompressible systems with the same 
time-dependent acceleration $\mathrm{g}(\mathrm{t})$.

We now summarize the specific results of Eqs. (57)-(64) applied to ideal (high Mach number) blast waves. We first treat the non-divergent (planar) case and then proceed to consider the effects of divergence on the model. With $d=1$, Eq. (58) becomes

$$
C_{2}=\frac{\Delta h_{i}}{L_{i}}\left(\frac{t_{i+1}}{t_{i}}\right)^{\frac{2}{\gamma(d+2)}}+\sigma^{-1} \sqrt{\frac{r_{0}}{L_{i}}}\left(\frac{t_{i+1}}{t_{i}}\right)^{\frac{1}{\gamma(d+2)}}\left[\left(\frac{t_{i+1}}{t_{i}}\right)^{\frac{1}{\gamma(d+2)}}-1\right], \quad \mathrm{d}=1
$$

where we have defined the parameter

$$
\sigma^{-1} \equiv 2(\kappa-1) \sqrt{\frac{|1-\eta|}{C_{D}}\left(\frac{\gamma(d+2)}{2}-1\right)} \text {. }
$$

Equation (66) is a quadratic in $\left(t_{i+1} / t_{i}\right)^{1 /[\gamma(d+2)]}$ that can be solved to yield

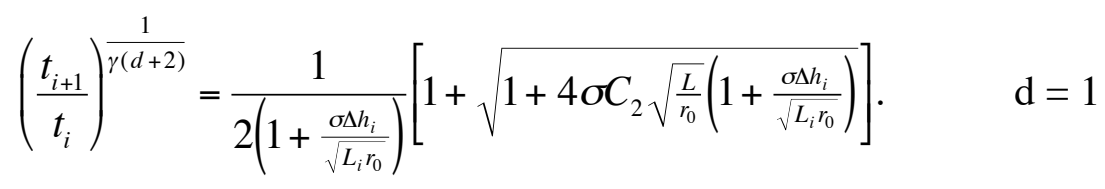

If $\Delta \mathrm{h}_{\mathrm{i}}$ is zero or otherwise negligible, then we have the somewhat simpler result

$$
\left(\frac{t_{i+1}}{t_{i}}\right)^{\frac{1}{\gamma(d+2)}}=\frac{1}{2}\left[1+\sqrt{1+4 \sigma C_{2} \sqrt{\frac{L_{i}}{r_{0}}}}\right]=\frac{1}{2}\left[1+\sqrt{1+4 \sigma C_{2} \mu^{i / 2} \sqrt{\frac{L_{0}}{r_{0}}}}\right] . \quad \mathrm{d}=1
$$

Together with the Eq. (61), Eq. (68) allows us to determine in what regions of parameter space loss of memory of the initial transverse scale might be possible. The sum in (61) has the necessary properties for loss of initial conditions as long as the inequality $4 \sigma C_{2} \mu^{i / 2} \sqrt{\frac{L_{0}}{r_{0}}}<<1 \Rightarrow \mu^{i}<<\frac{1}{4} \frac{1-\eta}{C_{D}}(\kappa-1)^{2} \frac{r_{0}}{L_{0}}$ is satisfied. The generalization of Eq. (23) to arbitrary $\mu$ suggests that, for classical RT, memory loss of initial conditions requires that $\mu^{N} \sim 100$, or $L=100 L_{0}$. This suggests that the above inequality is satisfied if $\frac{L_{0}}{r_{0}} \ll<\frac{1}{400} \frac{1-\eta}{C_{D}}(\kappa-1)^{2}$. In this case, a perturbation expansion of Eq. (68) gives 
$t_{i+1} / t_{i} \approx 1+\frac{\gamma(d+2) \sigma C_{2}}{\sqrt{\mu}-1}\left(\sqrt{L_{i} / r_{0}}-\sqrt{L_{0} / r_{0}}\right)$, which makes the sum in (61) independent of $\mathrm{L}_{0}$ when $\mathrm{L}_{\mathrm{N}} / \mathrm{r}_{0}$ becomes large compared to one. However, because our truncation of the perturbation expansion requires that $\mathrm{L}_{\mathrm{N}} / \mathrm{r}_{0}$ is small, we cannot conclude that memory of the initial transverse scale is lost for planar blast waves even if the initial scale is very small compared to $r_{0}$.

In general, the temporal evolution of the transverse scale can be determined by numerically solving the expression

$$
t_{N}=t_{0} \frac{t_{1}}{t_{0}} \ldots \frac{t_{N}}{t_{N-1}}=\frac{t_{0}}{2^{N}} \prod_{i=0}^{N-1}\left[1+\sqrt{1+4 \sigma C_{2} \mu^{i / 2} \sqrt{\frac{L_{0}}{r_{0}}}}\right] \quad \mathrm{d}=1
$$

for $N(t)$ and then evaluating $L(t)=L\left(t_{N}\right)=L_{0} \mu^{N(t)}$. Similarly, the evolution of the bubble height determined from

$$
h_{N}=h_{0} \frac{h_{1}}{h_{0}} \ldots \frac{h_{N}}{h_{N-1}}
$$

where, from Eq. (53), we have

$$
\frac{h_{i+1}}{h_{i}}=\left(\frac{t_{i+1}}{t_{i}}\right)^{\frac{2}{\gamma(d+2)}}\left[1+\frac{2 r_{0}}{h_{i}} \mu^{i / 2} \sqrt{\frac{L_{0}}{r_{0}} \frac{\mid 1-\eta}{C_{D}}\left(\frac{\gamma(d+2)}{2}-1\right)}\left(1-\left(\frac{t_{i}}{t_{i+1}}\right)^{\frac{1}{\gamma(d+2)}}\right)\right] . \quad \mathrm{d}=1
$$

The ratio $t_{i+1} / t_{i}$ becomes large compared to unity after many generations of bubble merger, at which point

$$
\frac{h_{i+1}}{h_{i}}>\left(\frac{t_{i+1}}{t_{i}}\right)^{\frac{2}{\gamma(d+2)}} \cdot \quad \mathrm{d}=1
$$

while $L_{i+1} / L_{i}=\mu$ for all $\mathrm{i}$. Asymptotically, then, the ratio $\mathrm{L}(\mathrm{t}) / \mathrm{h}(\mathrm{t})$ (the similarity parameter) is bounded by 


$$
\begin{gathered}
\frac{L_{N}}{h_{N}}=\frac{L_{N}}{L_{N-1}} \frac{h_{N-1}}{h_{N}} \frac{L_{N-1}}{h_{N-1}}<\mu\left(\frac{t_{N}}{t_{N-1}}\right)^{\frac{-2}{\gamma(d+2)}} \frac{L_{N-1}}{h_{N-1}} \\
<\mu^{N}\left(\frac{t_{N}}{t_{N-1}} \ldots \frac{t_{1}}{t_{0}}\right)^{\frac{-2}{\gamma(d+2)}} \frac{L_{0}}{h_{0}}<\frac{L_{0}}{h_{0}}\left(4 \sigma C_{2} \sqrt{\frac{L_{0}}{r_{0}}} \mu^{(N-3) / 4}\right)^{-N}, \quad \mathrm{~d}=1
\end{gathered}
$$

where we have used Eqs. (59) and (68) in addition to (72). According to (73), the similarity parameter decreases with increasing merger generation (or increasing time) at large $\mathrm{N}$. Thus there is no memory loss of initial conditions in the $\mathrm{d}=1$ case and no selfsimilar regime in which the mix width grows in proportion to the characteristic transverse scale. However, numerical evaluation of the model shows that, after about two generations of bubble merger, the value of the similarity parameter is nearly independent of the initial conditions (see Fig. 6). We call the quasi-self-similar regime and note that there is a period after its establishment when the similarity parameter is of order unity and changes quite slowly. At very late times, $\mathrm{h} / \mathrm{L}$ falls off like $\left(\mathrm{t} / \mathrm{t}_{0}\right)^{-\beta / 2}$. During this asymptotic phase, the bubble merger time has become very long and the instability evolution is dominated by amplitude stretching due to decompression.

Unless the Mach number of the incident blast wave is very high, however, it is unlikely that the $\mathrm{h} / \mathrm{L} \sim\left(\mathrm{t} / \mathrm{t}_{0}\right)^{-\beta / 2}$ state will be reached before the strong shock approximation is violated and the driving deceleration disappears (see Fig. 3). Because of this limitation, there is a maximum scale, determined by the initial conditions, that can be generated at a given interface. Thus the drive imposes an "effective box size" on the system that may or may not be smaller than the actual physical or computational box size.

In the divergent case, the merger time is determined by

$$
C_{2}=\frac{\Delta h_{i}}{L_{i}}+\frac{\sigma^{-1}}{\gamma(d+2)} \sqrt{\frac{r_{0}}{L_{i}}} \ln \left(\frac{t_{i+1}}{t_{i}}\right), \quad \mathrm{d}>1
$$


from which it is easy to obtain

$$
\frac{t_{i+1}}{t_{i}}=\exp \left\{\gamma(d+2) \sigma\left(C_{2}-\frac{\Delta h_{0}}{L_{i}}\right) \sqrt{\frac{L_{i}}{r_{0}}}\right\} \rightarrow \exp \left\{\gamma(d+2) \sigma C_{2} \sqrt{\frac{L_{i}}{r_{0}}}\right\} . \quad \mathrm{d}>1
$$

From Eq. (62) evaluated for $\mathrm{d}>1$ with the blast-wave-driven interface deceleration Eq. (42), we have

$$
m(t)=\left[\frac{1}{\sqrt{2 \pi} \gamma(d+2) C_{2} \sigma}\left(1-\frac{1}{\sqrt{\mu}}\right) \ln \left(\frac{t}{t_{0}}\right)+\frac{1}{\sqrt{\mu m_{0}}}\right]^{-2} \cdot \quad \mathrm{d}>1
$$

At late times, the mode number is independent of the initial conditions, as is the transverse scale

$$
\frac{L(t)}{r_{0}}=\left[\frac{1}{\gamma(d+2) C_{2} \sigma}\left(1-\frac{1}{\sqrt{\mu}}\right) \ln \left(\frac{t}{t_{0}}\right)+\sqrt{\frac{L_{0}}{r_{0} \mu}}\right]^{2}\left(\frac{t}{t_{0}}\right)^{\frac{2}{\gamma(d+2)}} \cdot \quad \mathrm{d}>1
$$

Inserting the time-dependent transverse scale into Eq. (57), we find that the mix width grows according to $\quad d>1$

$$
\frac{h(t)}{r_{0}}=\left\{\frac{h_{0}}{r_{0}}+\frac{1}{\gamma(d+2) \sigma(\kappa-1)}\left[\frac{1}{2 \sqrt{2 \pi} \gamma(d+2) C_{2} \sigma}\left(1-\frac{1}{\sqrt{\mu}}\right) \ln \left(\frac{t}{t_{0}}\right)+\sqrt{\frac{L_{0}}{r_{0} \mu}}\right] \ln \left(\frac{t}{t_{0}}\right)\right\}\left(\frac{t}{t_{0}}\right)^{\frac{2}{\gamma(d+2)}}
$$

Recalling the limiting time $t_{m}=t_{0}\left(\frac{2}{d+2} M_{0}^{a v e}\right)^{(d+2) / d}$ for a blast wave, we find that there is a minimum mode number $\mathrm{m}_{\text {lim }}$ that can be generated in a given divergent system, corresponding to a maximum transverse scale:

$$
m_{\lim }=2 \pi\left[\frac{1}{\gamma d C_{2} \sigma}\left(1-\frac{1}{\sqrt{\mu}}\right) \ln \left(\frac{2}{d+2} M_{0}^{a v e}\right)+\sqrt{\frac{2 \pi}{\mu m_{0}}}\right]^{-2} \cdot \quad \mathrm{d}>1
$$

For very high Mach numbers and/or very high initial mode numbers, the limiting mode is independent of the initial mode number (see Fig. 7a). Furthermore, the dependence of $\mathrm{m}_{\lim }$ on the initial Mach number (logarithmic squared) is fairly weak 
within the range of reasonable Mach numbers for strong blast waves (see Fig. 7b,c). Above about $\mathrm{M}_{0}^{\text {ave }}=20$, the $\mathrm{m}_{\text {lim }}$ varies by a factor of only a few when the Mach number varies by an order of magnitude.

Fryxell et al. have performed simulations of SN187A in which the unstable metal/He and $\mathrm{He} / \mathrm{H}$ interfaces are seeded by random grid-scale velocity perturbations behind the shock front. ${ }^{53}$ As the resolution of the calculations is varied, so too is the perturbation wavelength. At low resolution, corresponding to low initial mode number, the perturbation growth depends strongly on the resolution. At higher resolution, corresponding to a higher initial mode number of about $75-150$, the authors note that a "preferred" mode number in the range of 16-20 emerges at late times [see Fig. 8(a)]. In comparing with the merger model, we must choose an appropriate incident mach number $\mathrm{M}_{0}{ }^{\text {ave }}$. The Mach number of the shock is actually relatively low before shock breakout into the stellar atmosphere. It is only a few in the deep interior and climbs to about $\mathrm{M} \sim$ $10-20$ at the $\mathrm{He} / \mathrm{H}$ interface. ${ }^{53}$ After breakout, the Mach number is of order $10^{2}$. Since this higher value determines the lifetime of the blast wave, we take $\mathrm{M}_{0}{ }^{\text {ave }} \sim 100$. This suggests that memory of the initial mode number is likely to be retained until $t_{m}$ unless $m_{0}$ is greater than about 100 [see Fig. 4(a)]. At $\mathrm{M}_{0}^{\text {ave }} \sim 100$, the model predicts a limiting (minimum) mode number $\mathrm{m}_{\mathrm{lim}} \sim 20-30$, which is comparable to the preferred mode number $\mathrm{m} \sim 16-20$ found in the simulations.

We have performed a $\mathrm{CALE}^{54}$ simulation of a hypothetical laser-driven experiment that exhibits behavior very similar to the SN calculations of Fryxell et al. ${ }^{53} \mathrm{In}$ the simulation [see Fig. 8(b)], we assume that a laser has been used to heat the interior of a $50 \mathrm{mg} / \mathrm{cc}, 2.5 \mathrm{~mm}$ outer diameter, $0.75 \mathrm{~mm}$-thick foam shell to $200 \mathrm{eV}$. A high Mach 
number spherical blast wave is driven through the shell and into the surrounding $10^{-5} \mathrm{~g} / \mathrm{cc}$ air, driving the foam/air interface RT unstable. No perturbation is pre-imposed, but a grid-generated perturbation with $\mathrm{m}_{0} \approx 100$ has appeared by $5 \mu \mathrm{s}$. After a limited merger period, a late-time mode $\approx 24$ emerges in the freeze-out stage at about $20 \mu$ s.

Recent simulations aimed at studying $\mathrm{RT}$ at the $(\mathrm{C}+\mathrm{O}) / \mathrm{He}$ layer within corecollapse supernovae suggest that modes as low as $\mathrm{m}_{0} \approx 24$ may be present with significant amplitudes at the pre-shock interface due to neutrino-driven convection [see Fig. 8(c)]. ${ }^{27}$ According to Eq. (79), there should likely be no significant generation of larger scales in such a system. In fact, the simulations show that the late-time interface structure is dominated by the low-m modes present in the initial conditions.

Experimental observations of late-time modal structure in RT-unstable, divergent, blast-wave-driven systems, is rather limited, and we note here only two examples. First of all, $\mathrm{x}$-ray images of the Cassiopeia A core-collapse supernova remnant, ${ }^{55}$ obtained via the Chandra X-Ray Observatory, ${ }^{56}$ do appear to show spikes of core material protruding out from the explosion center [see Fig. 8(d)]. The explosion does not appear to have been completely isotropic, and the spikes are not uniform in angular position or transverse scale. Nevertheless, there are several large spikes visible in one quadrant with transverse scale that corresponds to a mode number $\mathrm{m} \sim 20$, similar to that seen in both the supernova and laser experiment simulations. Similarly, film footage of high-altitude nuclear detonations shows asymptotic modal structure that suggests $\mathrm{m} \sim 18-36$ and is reminiscent of supernova simulations [see Fig. 8(e)]. ${ }^{57,58}$ In both cases, the observed minimum mode number is in the range of limiting mode numbers predicted by the model for high incident Mach number and high initial mode number. Alternatively, the late-time 
structure could result from low modes $(\mathrm{m}<100)$ of significant amplitude present in the initial conditions.

\section{Statistical-mechanical model for blast-wave-driven case}

The principle value of the simple two-bubble-size merger model of Section $\mathrm{V}$ is that, despite its simplicity, it appears to qualitatively capture several of the important aspects of multimode, nonlinear, blast-wave-driven RT. More quantitative results can be achieved by applying the same expressions for the asymptotic bubble velocity [Eq. (52)], merger time [Eqs. (58), (68), and (75)], and corresponding amplitude increments [Eqs. (57), (71), and (78)], to a distribution of bubble sizes. This allows us to follow the modelpredicted evolution of the bubble-size distribution function $g(L, t)$, which is the solution to the statistical mechanical merger model equation [Eq. (4)]. Results of the statistical model represent for more sensible comparisons with experiments and simulations. In Fig. 9, we show the time-dependence of the similarity parameter $\langle\mathrm{L}(\mathrm{t})>/ \mathrm{h}(\mathrm{t})$ for the planar case as predicted by the statistical model (shown in red), where $<\mathrm{L}(\mathrm{t})\rangle$ is the bubble size expectation value of $g(L, t)$ (the average bubble size), and $h(t)$ remains the extent of the mix region. For the initial conditions, bubbles are selected at random from a uniform probability distribution from zero to $0.05 \mathrm{~L}_{\mathrm{box}}$, where $\mathrm{L}_{\mathrm{box}}$ is the box size. There are then about 40 bubbles at time zero, and their initial amplitudes are all set equal to $r_{0} / 200$. Because the $<\mathrm{L}(\mathrm{t})>$ contains contributions from smaller as well as dominant bubbles while $\mathrm{h}(\mathrm{t})$ is set by the largest bubbles, the similarity parameter is lower by a factor of a few than the prediction of the two-bubble-size model (compare Fig. 6). This lower value 
of $\left\langle\mathrm{L}(\mathrm{t})>/ \mathrm{h}(\mathrm{t})=0.2-0.4\right.$ for $\mathrm{t} / \mathrm{t}_{0}=10-100$ is in good agreement with Raptor simulations (black curves in Fig. 9), which give $<L(t)>/ h(t) \approx 0.1-0.4$ for the same $t / t_{0}$ and with analogous initial conditions. ${ }^{52}$ The simulations use initial spectra of various shapes (uniform, gaussian, $\mathrm{k}^{-1}$, and $\mathrm{k}^{-2}$ ) that all include modes of the same size relative to the computational box as in Fig. 9a. The drive used in the simulations is from the same planar laser-driven experiments described in Sec. IV. The agreement between the model and the simulations is particularly noteworthy in light of the fact that the interface motion in the simulations deviates somewhat from the ideal planar blast-wave-drive assumed in the model application.

\section{Conclusions}

For blast-wave-driven RT, self-similarity and loss of initial conditions are violated in the planar case but preserved in higher dimensions where divergence is present. The reason for this result is that in divergent systems the stretching factor for the transverse scales is the same as that of the parallel scales. Behind a planar blast wave, parallel scales are stretched in the rarefaction fan while transverse scales remain unaffected. Consequently, $\mathrm{L}(\mathrm{t}) / \mathrm{h}(\mathrm{t})$ decays asymptotically rather than approaching a constant value. After an early-time transient, the establishment of a quasi-self-similar regime is possible in systems driven by planar blast waves. Though time-dependent, the similarity parameter is nearly independent of the initial conditions during the quasi-selfsimilar regime. In addition, there is a period after the establishment of quasi-self- 
similarity but before the driving acceleration dies away during which the similarity parameter is of order unity and changes slowly in time.

The finite duration of the blast-wave drive sets a maximum scale that can be generated on a given interface. For divergent systems, this corresponds to a minimum mode number that depends weakly on the incident Mach number and initial mode number as long as both are sufficiently high. Self-similarity and loss of memory of initial conditions are in principle possible for divergent systems, but only for high initial characteristic mode numbers and high incident Mach numbers. This requirement has serious implications for supernovae. Initial conditions predicted by recent stellar calculations ${ }^{27.28}$ suggest that initial mode numbers present in supernova progenitors are not high enough to reach the self-similar regime. If these predictions are correct, the latetime interface structure observed in supernova remnants likely depends strongly on the initial conditions present within the star at the time of explosion.

\section{Acknowledgements}

This work was performed under the auspices of the U.S. Department of Energy by the University of California, Lawrence Livermore National Laboratory under contract No. W-7405-Eng-48. 


\section{References}

${ }^{1}$ J. W. S. Rayleigh, Scientific Papers (University press, Cambridge, 1899).

${ }^{2}$ G. I. Taylor, Proc. R. Soc. London, Ser. A 201, 192 (1950).

${ }^{3}$ S. Chandrasekhar, Hydrodynamic and hydromagnetic stability, (Dover Publications, New York, 1981).

${ }^{4}$ John D. Lindl, Inertial Confinement Fusion: the quest for ignition and high-energy gain, (Springer-Verlag, New York, 1998).

${ }^{5}$ Dov Shvarts, Oren Sadot, Dan Oron, Avi Rikanati, and Uri Alon G. BenDor, in Handbook of Shock Waves, edited by Gabi Ben-Dor, Ozer Igra, and Tov Elperin (Academic Press, London, 2001), Vol. 2, Chap. 14, pp. 489-543.

${ }^{6}$ H. J. Kull, Phys. Rep. 206, 197 (1991).

${ }^{7}$ M. N. Rosenbluth and C.L. Longmire, Ann. Physics, 26, 2227 (1957).

${ }^{8}$ R. Chevalier, Ap. J. 207, 872 (1976).

${ }^{9}$ Ya. B. Zel'dovich and Yu. P. Raizer, Physics of Shock Waves and High-Temperature Hydrodynamic Phenomema, (Dover Publications, New York 2002).

${ }^{10}$ National Research Council of the National Academies, Frontiers in High Energy Density Physics, (The National Academies Press, Washington, DC 2002).

${ }^{11}$ W. D. Arnett, J. N. Bachall, R. P. Kirshner, and S. E. Woosley, Ann. Rev. Astron. Astrophys. 27, 629 (1989).

${ }^{12}$ W. Hillebrandt and P. Hoflich, Rep. Prog. Phys. 52, 1421 (1989).

${ }^{13}$ D. Ryutov, R. P. Drake, J. Kane, E. Liang, B. A. Remington, and W. M. Wood-Vasey, Astrophys. J. 518, 821 (1999). 
${ }^{14}$ D. Arnett, B. Fryxell, E. Muller, Astrophys. J. 341, L63 (1989); E. Muller, B. Fryxell, D. Arnett, Astron. Astrophys. 251, 505 (1992)

${ }^{15}$ B. A. Remington, J. Kane, R. P. Drake, et al., Phys. Plasmas 4, 1994 (1997).

${ }^{16}$ K. S. Budil, B. A. Remington, T. A. Peyser, K. O. Mikaelian, P. L. Miller, et al., Phys. Rev. Lett., 76, 4536 (1996).

${ }^{17}$ J. Kane, D. Arnett, B. A. Remington, S. G. Glendinning, J. Castor, et al., Astrophys. J., 478, L75 (1997).

${ }^{18}$ J. Kane, D. Arnett, B. A. Remington, S. G. Glendinning, R. Wallace, et al., in Second Oak Ridge Symposium on Atomic and Nuclear Astrophysics, Oak Ridge, Tenessee, (1998).

${ }^{19}$ J. Kane, D. Arnett, B. A. Remington, S. G. Glendinning, G. Bazan, R. P. Drake, B. A. Fryxell, R. Teyssier, and K. Moore, Phys. Plasmas, 6(5), 2065 (1999).

${ }^{20}$ J. O. Kane, H. F. Robey, B. A. Remington, R. P. Drake, J. Knauer, et al., Phys. Rev. E, 63, 055401R (2001).

${ }^{21}$ H. F. Robey, J. O. Kane, B. A. Remington, R. P. Drake, O. A. Hurricane, et al., Phys. Plasmas, 8, 2446 (2001).

${ }^{22}$ R. P. Drake, H. F. Robey, O. A. Hurricane, Y. Zhang, B. A. Remington, et al., Astrophys. J., 564, 896 (2002).

${ }^{23}$ H. F. Robey, Y. Zhou, A. C. Buckingham, P. Keiter, B. A. Remington, et al., Phys. Plasmas, 10, 614 (2003).

${ }^{24}$ A. R. Miles, D. G. Braun, M. J. Edwards, H. F. Robey, R. P. Drake, et al., "Numerical Simulation of Supernova-Relevant Laser-driven Hydro Experiments on OMEGA," submitted to Phys. Plasmas. 
${ }^{25}$ K. I. Sharp, Physica D, 12, 3 (1984).

${ }^{26}$ J. Glimm and X. L. Li, Phys. Fluids, 31(8), 2077 (1988).

${ }^{27}$ K. Kifonidis, T. Plewa, H.-Th. Janka, and E. Muller, Astron. Astrophys., 408, 621 (2003).

${ }^{28}$ C. A. Meakin and W. D. Arnett, in preparation.

${ }^{29}$ D. Oron, L. Arazi, D. Kartoon, A. Rikanati, U. Alon, and D. Shvarts, Phys. Plasmas 8(6), 2883 (2001).

${ }^{30}$ J. C. V. Hanson, P. A. Rosen, T. J. Goldsack, K. Oades, P. Fieldhouse, N. Cowperthwaite, D. L. Youngs, N. Mawhinney, and A. J. Baxter, Laser Part. Beams 8, 51 (1990).

${ }^{31}$ G. Dimonte and M. Schneider, Phys. Rev. E 54, 3740 (1996).

${ }^{32}$ D. Layzer, Ap. J. 122, 1 (1955).

${ }^{33}$ R. M. Davies and G. I. Taylor, Proc. R. Soc. London Ser. A 200, 375 (1950).

${ }^{34}$ K. I. Read, Physica D, 12, 45 (1984).

${ }^{35}$ G. Glimm, J. Grove, and X. L. Li, in Proceedings of Taormina Conference, Sicily, 1987 (University of Mesina, Messina, Italy).

${ }^{36}$ U. Alon, D. Shvarts, and D. Mukamel, Phys. Rev. E, 48(2), 1008 (1993).

${ }^{37}$ U. Alon, J. Hecht, D. Mukamel, and D. Shvarts, Phys. Rev. Lett., 72(18), 2867 (1994).

${ }^{38}$ C. L. Gardner, J. Glimm, O. McBryan, R. Menikoff, D. H. Sharp, and Q. Zhang, Phys. Fluids 31, 447 (1998).

${ }^{39}$ J. Hecht, U. Alon, and D. Shvarts, Phys. Fluids 6, 4019 (1994).

${ }^{40}$ J. Glimm, X. L. Li, R. Menikoff, D. H. Sharp, and Q. Zhang, Phys. Fluids A 2(11), 2046 (1990). 
${ }^{41}$ D. L. Youngs, Physica D, 12, 32 (1984).

${ }^{42}$ J. Glimm, D. H. Sharp, Phys. Rev. Lett. 64(18), 2137 (1990).

${ }^{43}$ G. Dimonte and M. Schneider, Phys. Fluids 12(2), 304 (2000).

${ }^{44}$ L. I. Sedov, Prikl. Mat. Mekh. 10, 241 (1946).

${ }^{45}$ L. I. Sedov, Similarity and Dimensional Methods in Mechanics, (Academic Press, New York 1959).

${ }^{46}$ G. I. Taylor, Proc. R. Soc. London A201, 159 (1950).

${ }^{47}$ L. D. Landau and E. M. Lifshitz, Fluid Mechanics, (Pergamon Press, New York 1974).

${ }^{48}$ G. I. Barenblatt, Similarity, Self-Similarity and Intermediate Asymptotics, (Consultant Bureau, New York, 1979).

${ }^{49}$ R. D. Richtmyer, Commun. Pure Appl. Math. 13, 297 (1960).

${ }^{50}$ E. E. Meshkov, Izv. AN SSSR Mekhanika Zhidkosti I Gaza 4(5), 151 (1969).

${ }^{51}$ L. H. Howell, and J. A. Greenough, J. Comp. Phys., 184, 53 (2003).

${ }^{52}$ A. R. Miles, M. J. Edwards, and J. A. Greenough, "Effect of Initial Conditions on Compressible Rayleigh-Taylor Instability and Transition to Turbulence”, in preparation.

${ }^{53}$ B. Fryxell, E. Muller, and D. Arnett, Astrophys. J. 367, 619 (1991); D. Arnett, (private communication).

${ }^{54}$ R. T. Barton, Numerical Astrophysics (Jones and Bartlett, Boston, 1985).

${ }^{55}$ J. P. Hughes, C. E. Rakowski, D. N. Burrows, and P. O. Slane, Astrophys. J. 528, L109 (2000).

${ }^{56}$ M. C. Weisskopf, S. L. O’Dell, and L. P. van Speybroeck, Proc. SPIE, 2805, 2 (1996).

${ }^{57}$ Operation Fishbowl, produced by U.S. Department of Energy (distributed by U.S. 
Department of Energy, National Nuclear Security Administration, Nevada Site Office, Nuclear Testing Archive, M/S 400, P.O. Box 98521 Las Vegas, NV 89193-8521), VHS, black and white, $28 \mathrm{~min}$.

${ }^{58}$ Nukes in Space: the Rainbow Bombs, film produced by VCE inc. (distributed by VCE inc., http://www.vce.com/atomcentral.html, released 1999), DVD, color, 52 min. 


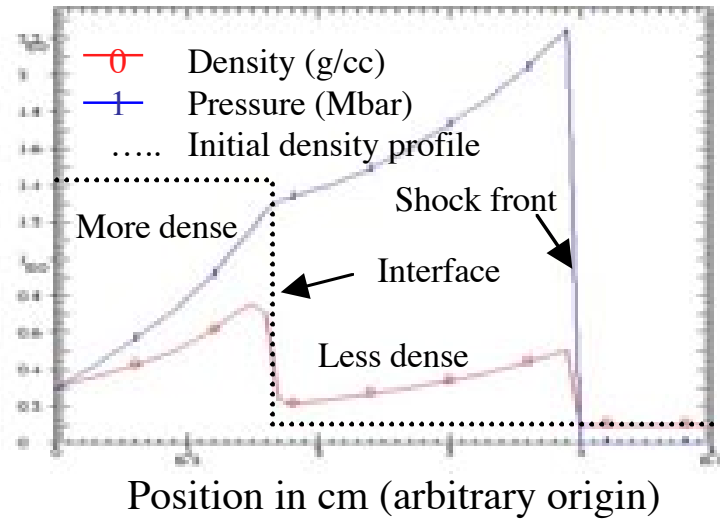

Figure 1: Density (curve 0) and pressure (curve 1) behind a blast wave that has passed through a material interface from a $1.42 \mathrm{~g} / \mathrm{cc}$ plastic to a $0.1 \mathrm{~g} / \mathrm{cc}$ foam. The interface is RT unstable due to the presence of antiparallel density and pressure gradients at the interface. The dotted line shows the initial (pre-shock) density profile. The data are from a 1D CALE simulation of the experiment-relevant planar hydrodynamics at $14 \mathrm{~ns}$. 


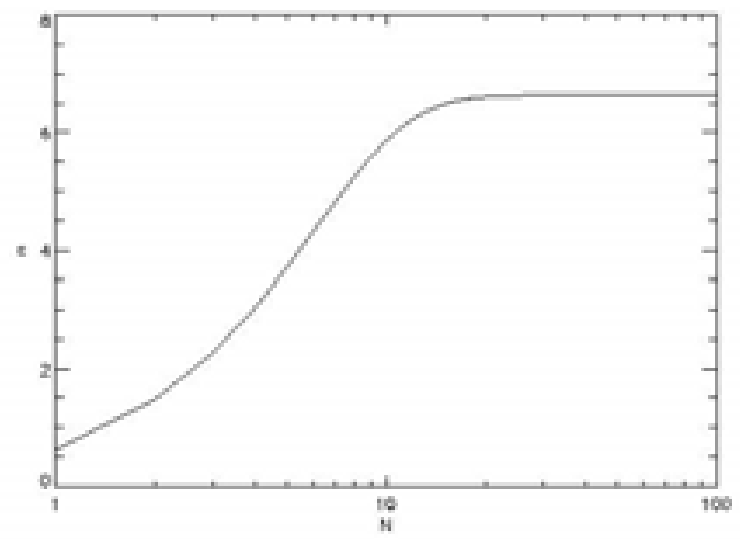

Figure 2: Loss of memory of the initial transverse scale: For a system having undergone $\mathrm{N}$ bubble merger generations, the number of generations $\mathrm{n}<\mathrm{N}$ necessary to include in the sum in Eq. (21) in order to reduce the error relative to the full sum (from 0 to $\mathrm{N}$ ) to $10 \%$. Above $\mathrm{N}=10$, the sum is dominated by the last seven terms. 


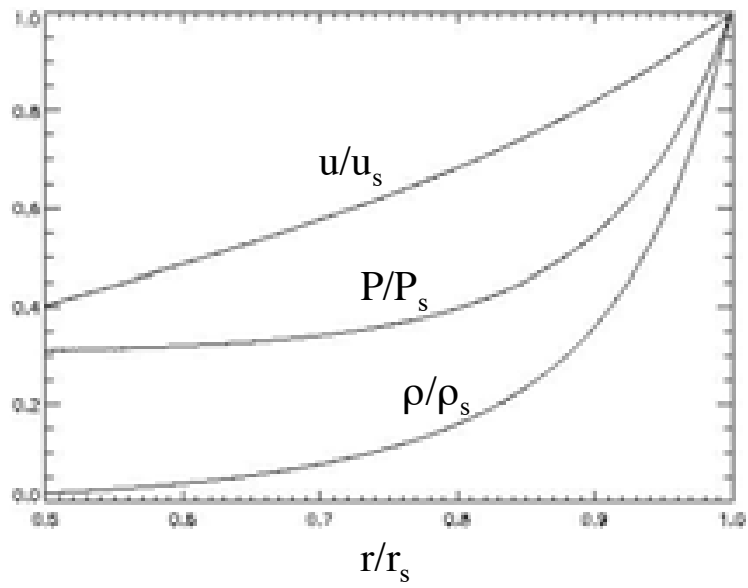

Figure 3: Typical solution (with $\gamma=5 / 3$ ) to the self-similar spherical blast wave problem. 


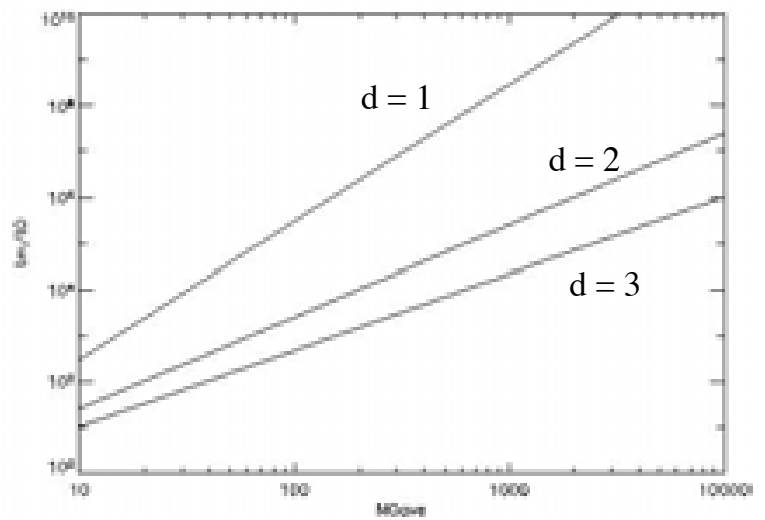

(a)

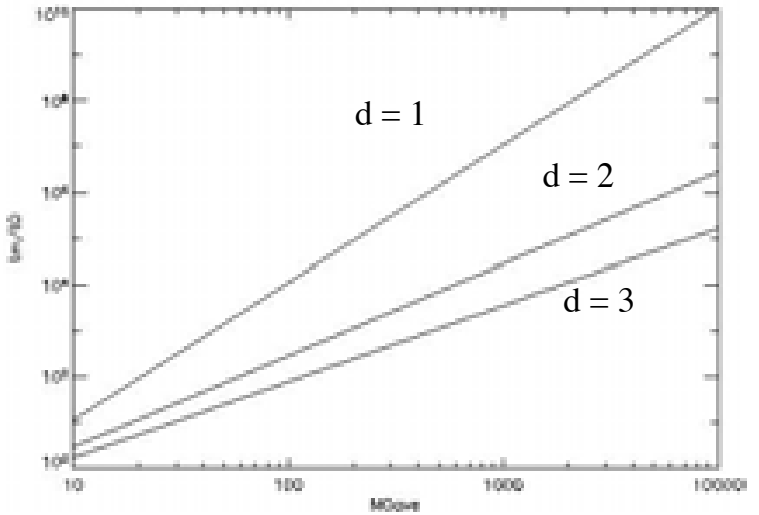

(b)

Figure 4: Estimated limiting drive time as a function of the average incident Mach number of the blast wave $\left[\mathrm{r}_{0} /\left(\mathrm{ct}_{0} /\right)\right]$. At $\mathrm{t}=\mathrm{t}_{\mathrm{m}}$, the Mach number predicted by the model in the strong shock approximation is equal to one in (a) and 3 in (b). 


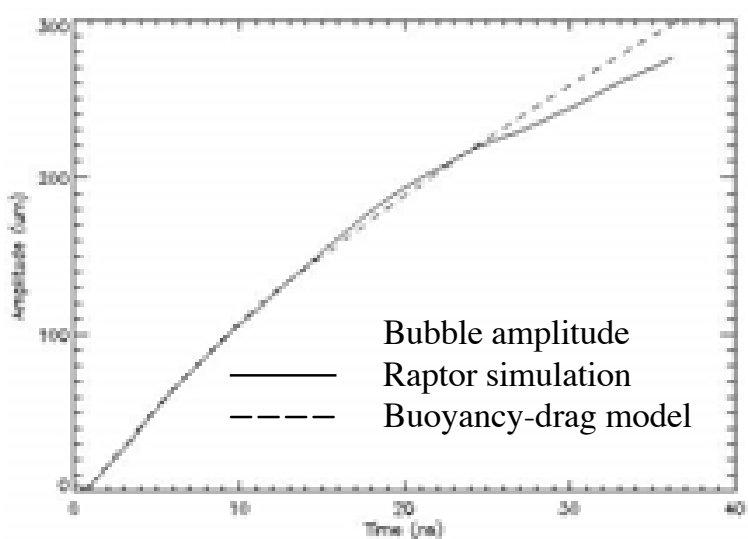

Figure 5: Application of generalized buoyancy-drag model (Eq. 57) to bubble amplitude growth in a simulation of a planar RT experiment in which a high-powered laser drives a plastic-foam interface with a pre-imposed single-mode perturbation. The drive resembles a1D blast wave with $\mathrm{M}_{0}$ ave $\approx 60$. The model curve (dashed line) with $\beta=0.51$ agrees very well with the simulation (solid line). 

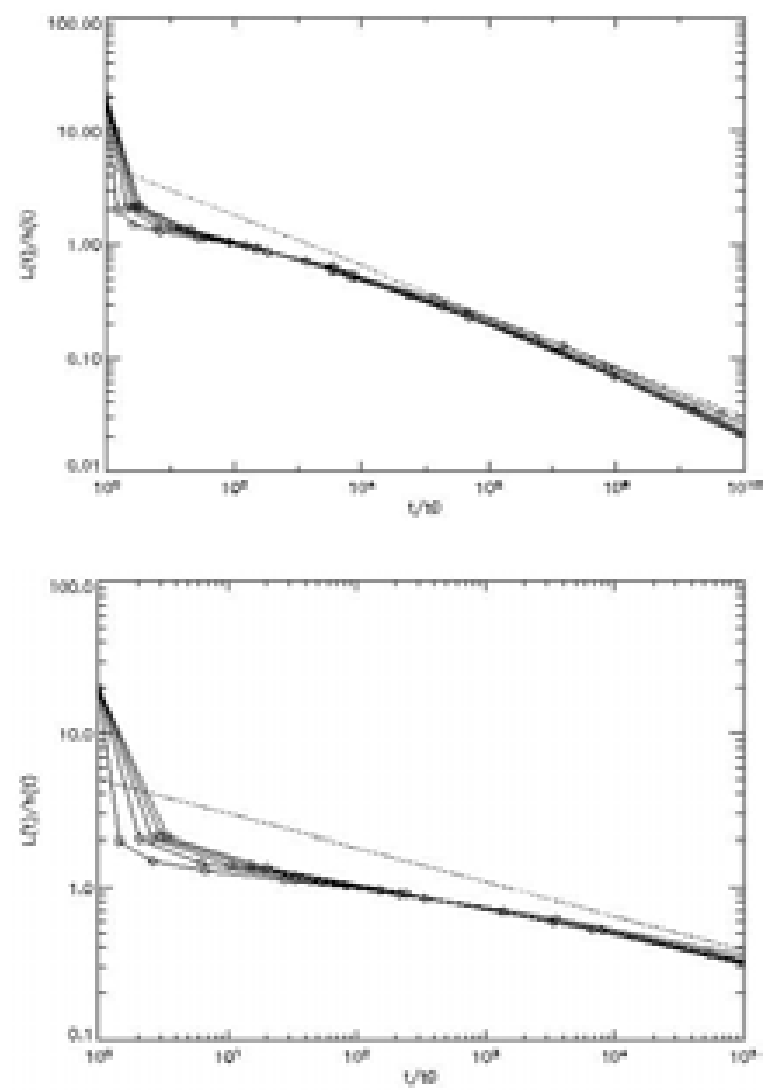

Figure 6: Quasi-self-similar regime: Rather than approaching a constant, the similarity parameter $\mathrm{h} / \mathrm{L}$ decays in time. However, the model predicts that, after about two generations of bubble merger, similarity parameter is independent of the initial conditions. In addition, the instability goes through a period of quasi-selfsimilar growth during which the similarity parameter changes quite slowly. At very late times, the similarity parameter scales like $\left(\mathrm{t} / \mathrm{t}_{0}\right)^{-\beta / 2}$. 


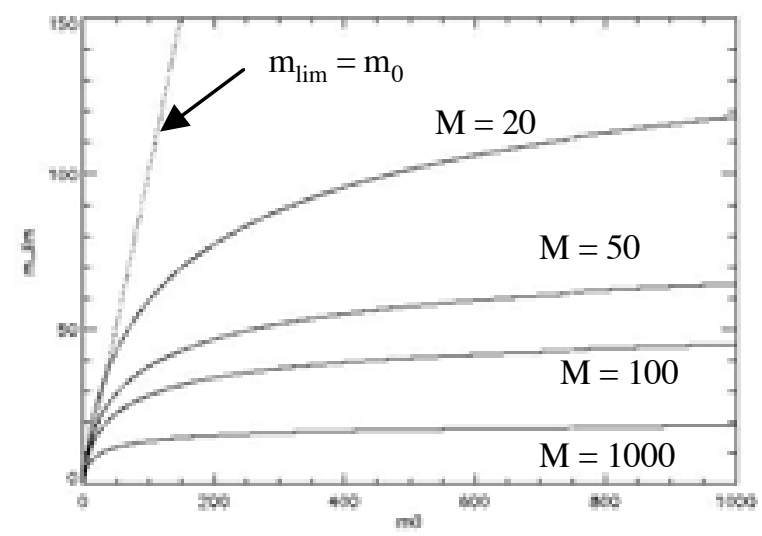

(a)

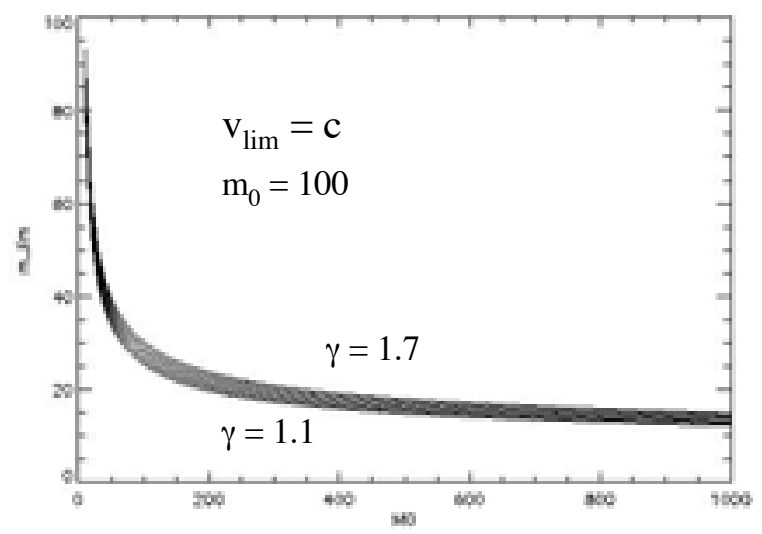

(b)

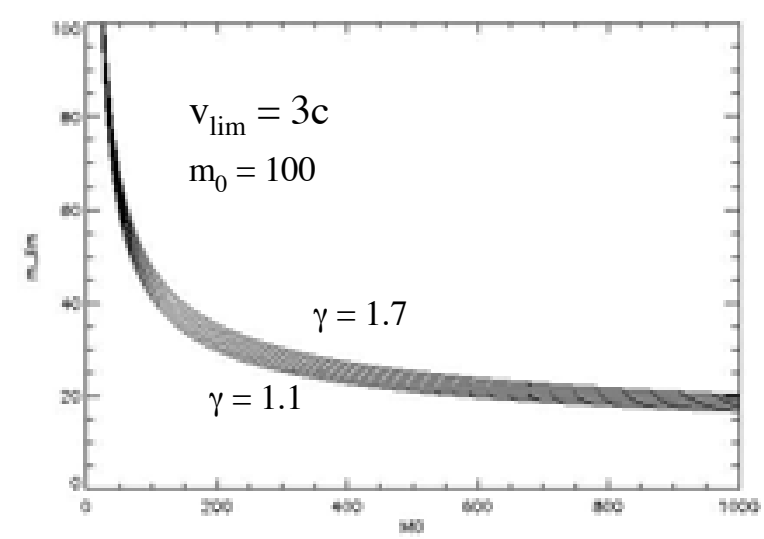

(c)

Figure 7: Minimum mode number in 3D-blast-wave-driven system. (a) For a given Mach number and high enough initial mode number, the dependence of $\mathrm{m}_{\mathrm{lim}}$ on the initial mode number is weak. [(b)-(c)] For a given initial mode number and high enough $\mathrm{M}_{0}$ ave , the minimum mode number depends weakly on the adiabatic index and the incident Mach number. In (a), $\mathrm{v}_{\lim }=\mathrm{c}$ and $\gamma=1.5$. 


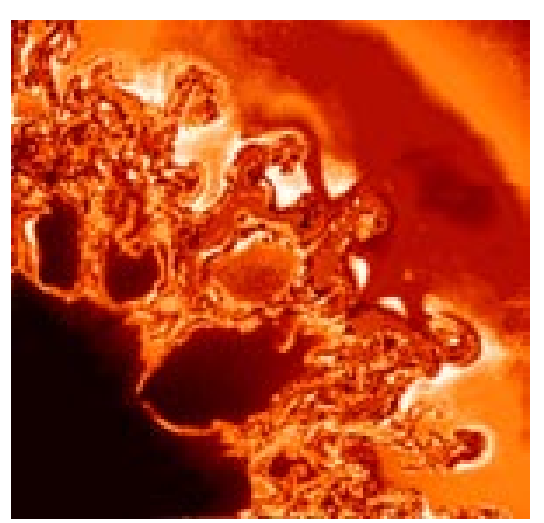

(a)
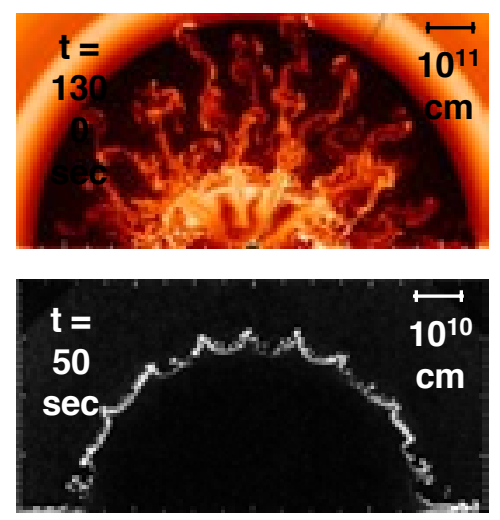

(c)

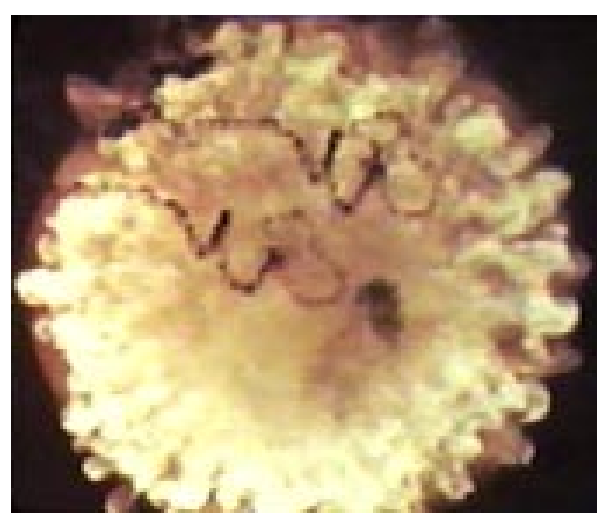

(e)
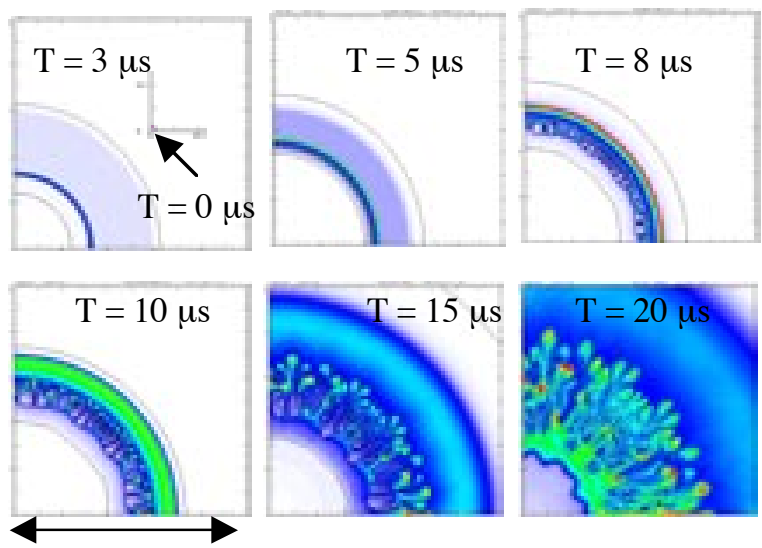

(b) $10 \mathrm{~cm}$

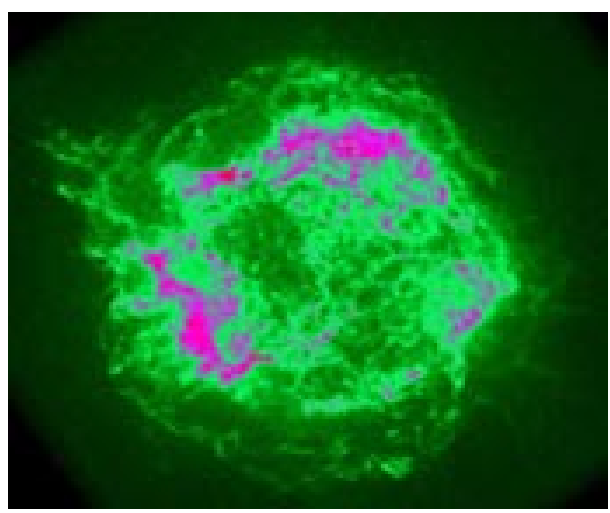

(d)

Figure 8: Similarities in asymptotic interface structure appear in a variety of systems driven by spherical blast waves. (a) Prometheus simulations of SN1987A with imposed grid-scale velocity perturbations behind the shock [Fryxell et al., Astrophys. J. 367, 619 (1991)] . When the initial mode number is high, a "preferred" mode number $\mathrm{m} \sim 16-20$ emerges at late times. (b) CALE simulation of a hypothetical laser experiment in which a spherical blast wave drives an RT-unstable foam/air interface. No perturbation is pre-imposed, but a grid-generated perturbation with $\mathrm{m}_{0} \approx 100$ has appeared by $5 \mu$ s. After a limited merger period, a late-time mode $\approx 24$ emerges in the freeze-out stage. (c) Recent SN simulations including neutrino-driven convection [Kifonidis et al., Astron. Astrophys. 408, 621 (2003)] show large-scale early-time perturbations that continue to dominate at late times. (d) X-ray images of the Cassiopeia A SNR [Hughes et al., Astrophys. J. 528, L109 (2000)] show large-scale structures corresponding to $m \sim 20$. (e) Images of high-altitude nuclear detonations [Peter Kuran, VCE inc.] show late-time freeze-out stage with $\mathrm{m} \sim 18-36$. 


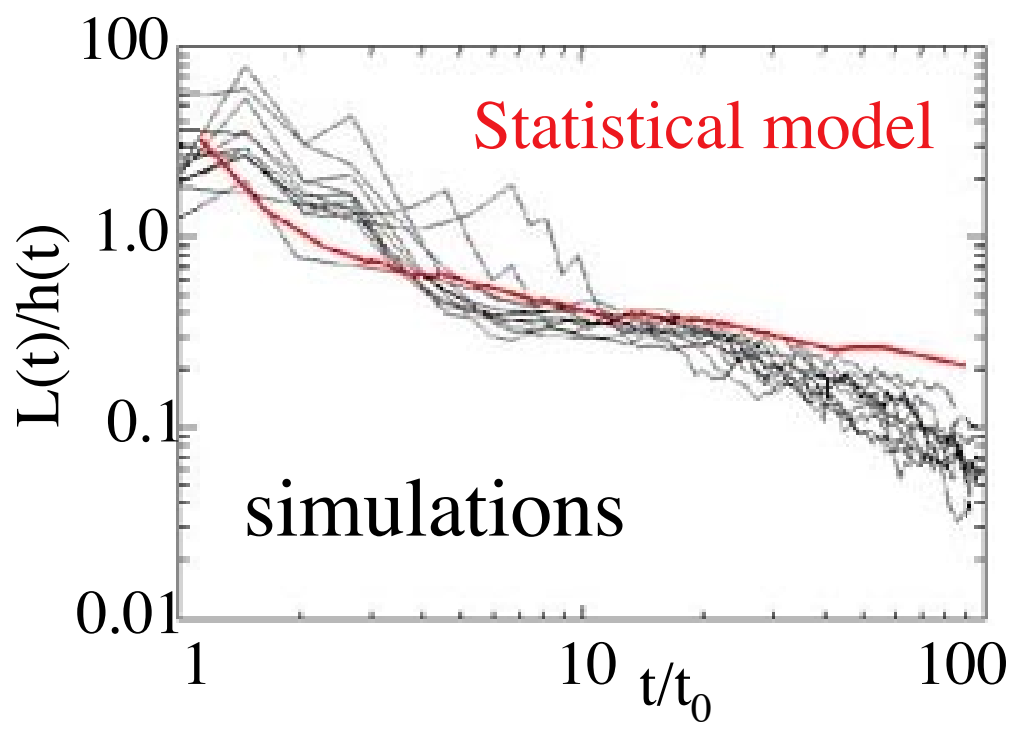

Figure 9: Time-dependence of the similarity parameter for the planar case. The prediction of the merger model applied to a broad distribution of bubble size is shown in red, and results from several 2D Raptor simulations with a variety of initial spectra are shown in black. The model-predicted value of the similarity is lower here than in the simpler two-bubble-size model of Section V (compare Fig. 6), but agrees well with the simulations. 


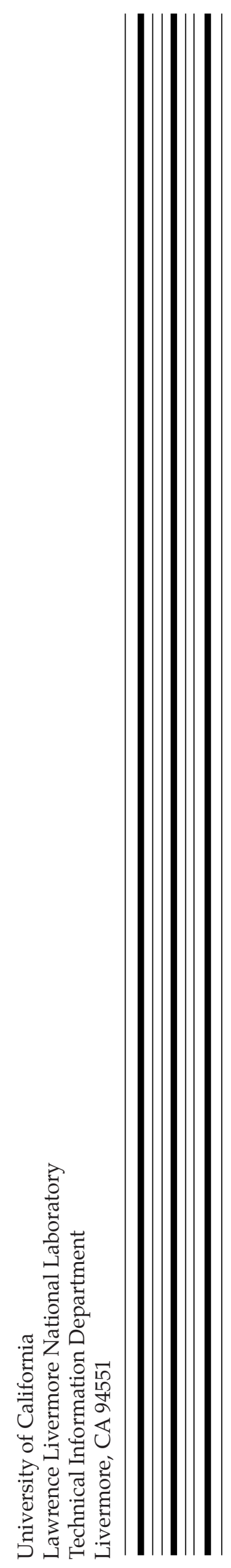

